Rev. Mat. Iberoamericana 23 (2007), no. 2, 481-512

\title{
On the regularity of averages over spheres for kinetic transport equations in hyperbolic Sobolev spaces
}

\section{Nikolaos Bournaveas and Susana Gutiérrez}

\begin{abstract}
We study the smoothing effect of averaging over spheres for solutions of kinetic transport equations in hyperbolic Sobolev spaces.
\end{abstract}

\section{Introduction}

In this paper we consider solutions $f: \mathbb{R}_{t} \times \mathbb{R}_{x}^{d} \times \mathbb{R}_{v}^{d} \rightarrow \mathbb{R}$ of the kinetic transport equation

$$
\partial_{t} f+v \cdot \nabla_{x} f=g,
$$

and study their averages over spheres

$$
\rho(t, x)=\int_{\mathbb{S}^{d-1}} f(t, x, v) d \sigma(v) .
$$

These averages come up in the radiative transfer equation which describes the scattering of photons in a hot medium $[1,2,16,25,8]$. Averages over balls of solutions of (1.1),

$$
\rho_{B}(t, x)=\int_{|v| \leq 1} f(t, x, v) d v,
$$

are known to be smoother than $f$, in all dimensions, by half a derivative. More precisely,

$$
\begin{aligned}
\|(1+|\tau| & +|\xi|)^{1 / 2} \widetilde{\rho_{B}}(\tau, \xi) \|_{L^{2}\left(\mathbb{R}^{1+d}\right)} \\
& \leq C(d)\left(\|f\|_{L^{2}\left(\mathbb{R}^{1+d \times B)}\right.}+\|g\|_{L^{2}\left(\mathbb{R}^{1+d} \times B\right)}\right)
\end{aligned}
$$

where $B:=\left\{v \in \mathbb{R}^{d}:|v| \leq 1\right\}$ and $\widetilde{\rho_{B}}$ denotes the space-time Fourier transform of $\rho_{B}$.

2000 Mathematics Subject Classification: Primary: 35B45 ; Secondary: 35F10.

Keywords: Averaging lemmas, transport equations, hyperbolic Sobolev spaces. 
The same gain of half a derivative is observed in all dimensions for averages over balls of solutions $f: \mathbb{R}_{x}^{d} \times \mathbb{R}_{v}^{d} \rightarrow \mathbb{R}$ of the stationary equation

$$
v \cdot \nabla_{x} f=g .
$$

These smoothing effects were first discovered in [18] and [17] and later developed in several papers $[4,7,9,10,11,12,13,15,19,20,21,22,23,26]$. See [24] for a review and an extended bibliography.

We now turn our attention to averages over spheres whose study was initiated in [6]. For solutions of the stationary equation (1.5) averages over spheres gain half a derivative in all dimensions, even in dimension $d=2$.

Proposition 1.1. Let $d \geq 2$. Let $f: \mathbb{R}_{x}^{d} \times \mathbb{R}_{v}^{d} \rightarrow \mathbb{R}$ be a solution of $(1.5)$ and define

$$
[f](x)=\int_{\mathbb{S}^{d-1}} f(x, v) d \sigma(v)
$$

Then

$$
\left\|(1+|\xi|)^{1 / 2} \widehat{[f]}(\xi)\right\|_{L^{2}\left(\mathbb{R}^{d}\right)} \leq C\left(\|f\|_{L^{2}\left(\mathbb{R}^{d} \times \mathbb{S}^{d-1}\right)}+\|g\|_{L^{2}\left(\mathbb{R}^{d} \times \mathbb{S}^{d-1}\right)}\right) .
$$

The proof uses the standard technique of [17] and is sketched in Section 2.

The situation is different for averages over spheres of solutions of the time-dependent equation (1.1). It is important to notice here that in the case of the stationary equation the ambient $x$-space has dimension $d$ and we average over $d-1$ dimensional spheres, while in the case of the timedependent equation the ambient $(t, x)$-space has dimension $d+1$ and we are again averaging over $d-1$ dimensional spheres. It was proved in [6] that in dimensions $d \geq 3$ averages over spheres gain half a derivative, exactly the same gain as for balls, although spheres have higher codimension. In dimension $d=2$ however averages over spheres only gain a quarter of a derivative. It was also shown in [6] that the loss of $1 / 4$ derivatives in two dimensions occurs near the characteristic cone $|\tau|=|\xi|$ in phase space and that we can recover the missing regularity by working in hyperbolic Sobolev spaces (see (1.10) below). Both in the case $d=2$ and in the case $d \geq 3$ these estimates for averages over spheres imply the classical estimate (1.4) for balls and in that sense they are stronger.

There is a simple geometric explanation for this discrepancy between two and higher dimensions. Suppose we can show that for some $\alpha \in(0,2)$ the following estimate holds, for all $\tau \in \mathbb{R}$, all unit vectors $\xi \in \mathbb{R}^{d}$ and all $\lambda>0$ :

$$
\sigma\left\{v \in \mathbb{S}^{d-1}:|\tau+v \cdot \xi|<\lambda\right\} \lesssim \lambda^{\alpha}\left({ }^{1}\right)
$$

\footnotetext{
${ }^{1} A \lesssim B$ means that there is a positive constant $C$, depending only on the dimension (and possibly on various other harmless parameters) such that $A \leq C B$.
} 
where $\sigma$ denotes the usual surface measure on $\mathbb{S}^{d-1}$. We can then show $\left(^{2}\right)$ that averages over spheres gain $\alpha / 2$ derivatives. To see how the measure of the set in estimate (1.8) behaves let us take $\xi=(0, \ldots, 0,1)$ and consider the following two extreme cases: if $\tau=0$ then the set becomes $\left\{v \in \mathbb{S}^{d-1}:\left|v_{d}\right|<\lambda\right\}$ and it is a strip around the equator of 'width' $\lambda$ whose surface measure is $\lesssim \lambda$. If $\tau= \pm 1$ then the set is $\left\{v \in \mathbb{S}^{d-1}:\left| \pm 1+v_{d}\right|<\lambda\right\}$ and it is a 'cap' centered at the South or North Pole whose surface measure is $\lesssim \lambda^{\frac{d-1}{2}}$. We may restrict our attention to small $\lambda$ 's. If $d \geq 3$ then $\frac{d-1}{2} \geq 1$ hence $\lambda^{\frac{d-1}{2}} \leq \lambda$ (the measure is concentrated near the equator). In this case $\alpha=1$ and the gain is $1 / 2$ derivatives. On the other hand, if $d=2$, then $\lambda \leq \lambda^{\frac{d-1}{2}}=\lambda^{\frac{1}{2}}$, so $\alpha=1 / 2$ and the gain is $1 / 4$ derivatives.

Let us now recall the main estimate of $[6]$ :

Theorem. ([6]) Let $f$ be a solution of (1.1). Then the average over the unit sphere defined in (1.2) satisfies the following estimates: If $d \geq 3$ then,

$$
\begin{aligned}
\|(1+|\tau| & +|\xi|)^{\frac{1}{2}} \tilde{\rho}(\tau, \xi) \|_{L^{2}\left(\mathbb{R}^{1+d}\right)} \\
& \leq C\left(\|f\|_{L^{2}\left(\mathbb{R}^{1+d} \times \mathbb{S}^{d-1}\right)}+\|g\|_{L^{2}\left(\mathbb{R}^{1+d} \times \mathbb{S}^{d-1}\right)}\right)
\end{aligned}
$$

If $d=2$ then,

$$
\begin{aligned}
\|(1+|\tau|+|\xi|)^{\frac{1}{4}}(1+\| \tau \mid & -|\xi| \mid)^{\frac{1}{4}} \tilde{\rho}(\tau, \xi) \|_{L^{2}\left(\mathbb{R}^{1+2}\right)} \\
& \leq C\left(\|f\|_{L^{2}\left(\mathbb{R}^{1+2} \times \mathbb{S}^{1}\right)}+\|g\|_{L^{2}\left(\mathbb{R}^{1+2} \times \mathbb{S}^{1}\right)}\right)
\end{aligned}
$$

In Section 6 we give a counterexample which shows that the pair $(s, \delta)=$ $(1 / 4,1 / 4)$ in two dimensions and the pair $(s, \delta)=(1 / 2,0)$ in three dimensions are best possible.

The geometric viewpoint discussed above suggests that there should be an improvement to these estimates in dimensions $d \geq 4$. Indeed, the measure in the left hand side of (1.8) behaves differently depending on the relation between $|\tau|$ and $|\xi|$, because it is this relation that determines the location of the set on the unit sphere. We expect better estimates when the set is near one of the Poles, and this happens when $(\tau, \xi)$ is 'near the light cone' $|\tau|=|\xi|$. This improvement will be expressed, as in [6], in hyperbolic Sobolev spaces. We shall prove in Section 3 the following

\footnotetext{
${ }^{2}$ The proof uses a standard technique from [17]. See also the presentation in [3]. With a little care in the proof we can actually replace $\|f\|+\|g\|$ in the right hand side by $\|f\|^{1-\frac{\alpha}{2}}\|g\|^{\frac{\alpha}{2}}$.
} 
Theorem 1. (Hyperbolic Sobolev regularity) Let $d \geq 2$ and $f$ solve (1.1). Let $s, \delta \in \mathbb{R}$ be such that:

$$
s+\delta \leq \frac{1}{2} \quad \text { and } \begin{cases}s \leq \min \left\{\frac{d-1}{4}, 1\right\}, & \text { if } \quad d \neq 5 \\ s<1, & \text { if } d=5\end{cases}
$$

Then the average $\rho$ defined by (1.2) satisfies the following estimate:

$$
\begin{aligned}
\left\|(1+|\tau|+|\xi|)^{s}(1+\| \tau|-| \xi||)^{\delta} \widetilde{\rho}(\tau, \xi)\right\|_{L^{2}\left(\mathbb{R}^{1+d}\right)} & \\
& \lesssim\|f\|_{L^{2}\left(\mathbb{R}^{1+d} \times \mathbb{S}^{d-1}\right)}+\|g\|_{L^{2}\left(\mathbb{R}^{1+d} \times \mathbb{S}^{d-1}\right)}
\end{aligned}
$$

Some remarks are in order. In all cases we would like $s$, the exponent of the 'good' weight $1+|\tau|+|\xi|$, to be as large as possible. For $d=2$ the best that (1.11) allows is $s=1 / 4$, and we can then take $\delta=1 / 4$. This recovers (1.10). For $d=3$ we can take $s=1 / 2$ and $\delta=0$ and this recovers (1.9). Let us examine more closely dimension $d=4\left({ }^{3}\right)$. We can take $s=3 / 4$ and $\delta=-1 / 4$. The estimate then is:

$$
\begin{aligned}
\left\|(1+|\tau|+|\xi|)^{3 / 4}(1+\| \tau|-| \xi||)^{-1 / 4} \widetilde{\rho}(\tau, \xi)\right\|_{L^{2}\left(\mathbb{R}^{1+4}\right)} & \\
& \lesssim\|f\|_{L^{2}\left(\mathbb{R}^{1+4} \times \mathbb{S}^{3}\right)}+\|g\|_{L^{2}\left(\mathbb{R}^{1+4} \times \mathbb{S}^{3}\right)}
\end{aligned}
$$

Observe first that $(1+|\tau|+|\xi|)^{3 / 4}(1+|| \tau|-| \xi||)^{-1 / 4} \geq(1+|\tau|+|\xi|)^{1 / 2}$ so (1.13) implies (1.9). However, (1.13) also shows that for $(\tau, \xi)$ with ||$\tau|-| \xi|| \lesssim 1$ ('near the cone') we can control $(1+|\tau|+|\xi|)^{3 / 4} \widetilde{\rho}(\tau, \xi)$ i.e. gain $3 / 4$ derivatives - an extra gain of $1 / 4$ derivatives over the classical $1 / 2$-gain. We have similar gains 'near the cone' in all dimensions $d \geq 4$. This is in sharp contrast to what happens in two dimensions where a loss of regularity occurs 'near the cone'.

In dimension $d=5$, condition (1.11) excludes the end point case $s=1$. Actually, we can take $s=1$ (and $\delta=-1 / 2$ ) if we allow for a logarithmic term in $1+|| \tau|-| \xi||$ (see $(1.15))$.

It was noted in [6] that in the 'elliptic' region $|\tau| \geq|\xi|$ we have an extra gain of regularity (see (19) and (20) in [6]). In the present paper estimate (1.12) will be a consequence of the estimates in the following Theorem which take into account this gain in the region $|\tau| \geq|\xi|$. In these estimates $x_{+}$denotes the positive part of the real number $x$.

\footnotetext{
${ }^{3}$ It turns out that one can use the corresponding pointwise four dimensional estimate to improve considerably estimates (50) and (51) of [6] (see Theorem 3 below and Remark 4.1 on p.498). The new estimates we present in this paper are best possible.
} 
Theorem 2. Let $d \geq 2$ and $f$ be a solution of the equation (1.1). Then, the average over the sphere in (1.2) satisfies the following estimates:

(i) If $d \neq 5$, define $s=1+\min \left\{\frac{d-5}{4}, 0\right\}$ and

$$
\delta_{1}=-\min \left\{\frac{d-5}{4}, 0\right\}, \delta_{2}=-\frac{1}{2}-\min \left\{\frac{d-5}{4}, 0\right\} .
$$

Then:

$$
\begin{aligned}
\|(1+|\tau| & +|\xi|)^{s}\left(1+(|\tau|-|\xi|)_{+}\right)^{\delta_{1}}\left(1+(|\xi|-|\tau|)_{+}\right)^{\delta_{2}} \widetilde{\rho}(\tau, \xi) \|_{L^{2}\left(\mathbb{R}^{1+d}\right)} \\
& \lesssim\|f\|_{L^{2}\left(\mathbb{R}^{1+d}, \mathbb{S}^{d-1}\right)}+\|g\|_{L^{2}\left(\mathbb{R}^{1+d}, \mathbb{S}^{d-1}\right)}
\end{aligned}
$$

(ii) If $d=5$,

$$
\begin{aligned}
& \left\|(1+|\tau|+|\xi|)\left(1+(|\xi|-|\tau|)_{+}\right)^{-\frac{1}{2}}\left(1+\log \left(\frac{1+|\tau|+|\xi|}{1+|| \tau|-| \xi||}\right)\right)^{-\frac{1}{2}} \widetilde{\rho}(\tau, \xi)\right\|_{L^{2}\left(\mathbb{R}^{1+5}\right)} \\
& \lesssim\|f\|_{L^{2}\left(\mathbb{R}^{1+5}, \mathbb{S}^{4}\right)}+\|g\|_{L^{2}\left(\mathbb{R}^{1+5}, \mathbb{S}^{4}\right)}
\end{aligned}
$$

The proof of this Theorem uses the methods of [6] together with a detailed study and sharp estimates for the integrals $J_{l}^{m}$ (see Proposition 3.1). In the region $|\tau| \geq|\xi|$ (inside the cone) we use estimates for the integrals $H_{b}^{a}$ (see Lemma 3.2) introduced by Foschi and Klainerman in [14] in connection with sharp bilinear null form estimates for solutions of the wave equation. To deal with the region $|\tau| \leq|\xi|$ we introduce and estimate the new integrals $F_{b}^{a}$ and $G_{b}^{a}$ (see Lemmata 3.3 and 3.4).

Estimates on averages of solutions of kinetic equations with right handsides which contain derivatives with respect to $v$ are used in the study of the Maxwell-Vlasov equations. In Section 4 we will study averages over spheres for the equation

$$
\partial_{t} f+v \cdot \nabla_{x} f=\Omega_{v}^{i, j} g
$$

where $\Omega_{v}^{i, j}=v_{i} \frac{\partial}{\partial v_{j}}-v_{j} \frac{\partial}{\partial v_{i}}$ are tangential derivatives. We restrict ourselves to tangential derivatives because we need to be able to integrate by parts on the sphere. Moreover, if we take the point of view that $f$ and $g$ are only defined on the sphere then only tangential derivatives make sense. In [6] it was shown that if $d \geq 3$ then $\rho$ gains $1 / 4$ derivatives (exactly the same gain as for balls), but gains only $1 / 8$ derivatives if $d=2$. The operators $\Omega^{i, j}$ introduce a special structure which is reminiscent of the null forms structure for the wave equation and allows better than expected estimates (see the Remarks in Section 4 of [6]). Taking this into account the following improvement to the 1/8 gain in two dimensions was established in [6]. 
Theorem. ([6]) Let $f$ solve equation (1.16) in $d=2$ dimensions and define $\rho$ by $(1.2)$. Let $(s, \delta)=\left(\frac{1}{7}, 0\right)$ or $\left(\frac{1}{16}, \frac{3}{16}\right)$. Then

$$
\begin{aligned}
& \left\|(1+\| \tau|-| \xi||)^{\delta}(1+|\tau|+|\xi|)^{s} \tilde{\rho}(\tau, \xi)\right\|_{L^{2}\left(\mathbb{R}^{1+2}\right)} \\
& \leq C\left(\|f\|_{L^{2}\left(\mathbb{R}^{1+2} \times \mathbb{S}^{1}\right)}+\|g\|_{L^{2}\left(\mathbb{R}^{1+2} \times \mathbb{S}^{1}\right)}\right)
\end{aligned}
$$

In this paper we improve these results to $(s, \delta)=\left(\frac{1}{6}, 0\right)$ and $(s, \delta)=$ $\left(\frac{1}{8}, \frac{1}{8}\right)$ and show that the new results are best possible. We shall prove the following

Theorem 3. Let $f, g: \mathbb{R}^{1+2} \times \mathbb{S}^{1} \longrightarrow \mathbb{R}$ satisfy

$$
\partial_{t} f+v \cdot \nabla_{x} f=\Omega_{v}^{i, j} g
$$

where $\Omega_{v}^{i, j}=v_{i} \frac{\partial}{\partial v_{j}}-v_{j} \frac{\partial}{\partial v_{i}}$. Then, the average $\rho$ defined in (1.2) satisfies:

$$
\left\|(1+|\tau|+|\xi|)^{\frac{1}{6}} \tilde{\rho}(\tau, \xi)\right\|_{L^{2}\left(\mathbb{R}^{1+2}\right)} \lesssim\left(\|f\|_{L^{2}\left(\mathbb{R}^{1+2} \times \mathbb{S}^{1}\right)}+\|g\|_{L^{2}\left(\mathbb{R}^{1+2} \times \mathbb{S}^{1}\right)}\right)
$$

and the hyperbolic Sobolev estimate:

$$
\begin{aligned}
\left\|(1+|\tau|+|\xi|)^{\frac{1}{8}}(1+\| \tau|-| \xi||)^{\frac{1}{8}} \tilde{\rho}(\tau, \xi)\right\|_{L^{2}\left(\mathbb{R}^{1+2}\right)} & \\
& \lesssim\left(\|f\|_{L^{2}\left(\mathbb{R}^{1+2} \times \mathbb{S}^{1}\right)}+\|g\|_{L^{2}\left(\mathbb{R}^{1+2} \times \mathbb{S}^{1}\right)}\right) .
\end{aligned}
$$

In Section 5 we study the Initial Value Problem

$$
\partial_{t} f+v \cdot \nabla_{x} f=0, \quad f(0)=f_{0}
$$

and prove averaging lemmas in hyperbolic Sobolev spaces in all dimensions. These estimates improve and generalize those of [6]. We present here a proof which works in all dimensions and also provides a pointwise estimate for $\tilde{\rho}(\tau, \xi)$ (see (5.5)) while the proof in [6] only gave an estimate for a certain weighted $\tau$-integral of $\tilde{\rho}(\tau, \xi)$ (see $(5.4))$. We shall prove the following

Theorem 4. Let $d \geq 2$ and $f$ be a solution of the IVP (5.2). Then the corresponding average $\rho$ satisfies

$$
\left\|(1+|\tau|+|\xi|)^{\frac{d-1}{4}}(1+\| \tau|-| \xi||)^{-\frac{d-3}{4}} \widetilde{\rho}(\tau, \xi)\right\|_{L^{2}\left(\mathbb{R}^{1+d}\right)} \lesssim\left\|f_{0}\right\|_{L^{2}\left(\mathbb{R}^{d} \times \mathbb{S}^{d-1}\right)} .
$$

Notation: We use $\hat{f}(\xi)$ to denote the Fourier transform of a function $f$ on $\mathbb{R}^{d}$ and $\widetilde{F}(\tau, \xi)$ to denote the space-time Fourier transform of a function $F$ on $\mathbb{R}^{d+1}$. For simplicity we assume that all initial data and right hand sides of our equations are smooth functions which decay sufficiently fast at infinity. Throughout the paper, $p \lesssim q$ means that $p \leq C q$ for some positive constant $C$ which may depend on the dimension. Also $p \approx q$ means $q \lesssim p \lesssim q$.

Acknowledgements: The authors acknowledge with pleasure several helpful discussions with Benoit Perthame and Luis Vega. 


\section{Averages over spheres for solutions of the stationary equation}

In this section we sketch the proof of Proposition 1.1 and show that the exponent $1 / 2$ is best possible.

Proof of Proposition 1.1. We have $\hat{f}(\xi, v)=\frac{\hat{h}(\xi, v)}{1+i v \cdot \xi}$, where $h=f+g$, therefore

$$
\widehat{[f]}(\xi)=\int_{\mathbb{S}^{d-1}} \frac{\hat{h}(\xi, v)}{1+i v \cdot \xi} d \sigma(v) .
$$

Applying the Cauchy-Schwarz inequality we get

$$
|\widehat{[f]}(\xi)| \leq\left(\int_{\mathbb{S}^{d-1}}|\hat{h}(\xi, v)|^{2} d \sigma(v)\right)^{1 / 2} J(\tau, \xi)
$$

where $J(\xi)^{2}=\int_{\mathbb{S}^{d-1}} \frac{d \sigma(v)}{1+(v \cdot \xi)^{2}}$, so it suffices to show that $J(\xi)^{2} \leq \frac{C}{1+|\xi|}$. This integral can easily be estimated in all dimensions as follows:

$$
\begin{aligned}
J(\xi)^{2} & \approx \int_{0}^{\pi} \frac{(\sin \theta)^{d-2}}{1+|\xi|^{2} \cos ^{2} \theta} d \theta \\
& \lesssim \int_{0}^{\pi} \frac{1}{1+|\xi|^{2} \cos ^{2} \theta} d \theta \\
& \approx \int_{0}^{1} \frac{1}{\left(1+|\xi|^{2} x^{2}\right) \sqrt{1-x^{2}}} d x \quad(x:=\cos \theta) \\
& \lesssim \int_{0}^{1 / 2} \frac{1}{1+|\xi|^{2} x^{2}} d x+\frac{1}{|\xi|^{2}} \int_{1 / 2}^{1} \frac{1}{\sqrt{1-x^{2}}} d x \\
& =\frac{1}{|\xi|} \int_{0}^{\frac{|\xi|}{2}} \frac{1}{1+y^{2}} d y+\frac{1}{|\xi|^{2}} \\
& \lesssim \frac{1}{|\xi|}
\end{aligned}
$$

This settles the case $|\xi| \geq 1$. The case $|\xi| \leq 1$ is trivial.

To see that the exponent $1 / 2$ in Proposition 1.1 is the best possible we observe that estimate (1.7) implies the corresponding estimate for averages over balls $\left({ }^{4}\right)$. If (1.7) was true with an exponent larger than $1 / 2$ then the same argument would show that averages on balls gained more than $1 / 2$ derivatives. Since $1 / 2$ is the best possible exponent for balls [23], it follows that it is the also the best possible exponent for spheres.

\footnotetext{
${ }^{4}$ write $\int_{|v| \leq 1} f(x, v) d v=\int_{0}^{1} \int_{\mathbb{S}_{r}^{d-1}} f(x, v) d \sigma(v) d r$ and use the analogue of (1.7) for the sphere of radius $r$.
} 


\section{Averages over spheres for $\partial_{t} f+v \cdot \nabla_{x} f=g$}

The proof of Theorem 2 relies on sharp estimates for the integrals

$$
J_{l}^{m}(\tau, \xi)=\int_{0}^{\pi} \frac{(\sin \theta)^{m}}{\left[1+(\tau+|\xi| \cos \theta)^{2}\right]^{l}} d \theta, \quad \tau \in \mathbb{R}, \xi \in \mathbb{R}^{d}
$$

Proposition 3.1. Let $m>-1, l>1 / 2$ and define $\alpha=\min \left\{\frac{m+1-4 l}{2}, 0\right\}$. Then the integrals $J_{l}^{m}(\tau, \xi)$ satisfy the following pointwise estimates:

(i). If $|\tau| \geq|\xi|$,

$$
J_{l}^{m}(\tau, \xi) \approx \begin{cases}\frac{(1+|\tau|-|\xi|)^{\alpha}}{(1+|\tau|+|\xi|)^{2 l+\alpha}}, & \text { if } m+1 \neq 4 l \\ \frac{1}{(1+|\tau|+|\xi|)^{2 l}}\left(1+\log \frac{1+|\tau|+|\xi|}{1+|\tau|-|\xi|}\right), & \text { if } \quad m+1=4 l\end{cases}
$$

(ii). If $|\tau| \leq|\xi|$,

$$
J_{l}^{m}(\tau, \xi) \lesssim \begin{cases}\frac{(1+|\xi|-|\tau|)^{2 l-1+\alpha}}{(1+|\xi|+|\tau|)^{2 l+\alpha}}, & \text { if } m+1 \neq 4 l \\ \frac{(1+|\xi|-|\tau|)^{2 l-1}}{(1+|\xi|+|\tau|)^{2 l}}\left(1+\log \frac{1+|\xi|+|\tau|}{1+|\xi|-|\tau|}\right), & \text { if } m+1=4 l\end{cases}
$$

Estimate (3.2) is sharp, except in a certain case explained in Remark 3.5 on page 494. In the next three Lemmata we collect some estimates that come up repeatedly in the proof of Proposition 3.1.

Lemma 3.2. Let $b>-1, a \in \mathbb{R}$. For $\lambda>0$ define

$$
H_{b}^{a}(\lambda)=\int_{0}^{1}(\lambda+t)^{a} t^{b} d t
$$

Then

$$
\begin{array}{ll}
H_{b}^{a}(\lambda) \approx \lambda^{a}, & \text { if } \lambda \gtrsim 1, \\
H_{b}^{a}(\lambda) \approx \lambda^{\min \{a+b+1,0\}}, & \text { if } 0<\lambda \lesssim 1, a+b+1 \neq 0, \\
H_{b}^{a}(\lambda) \approx 1+|\log \lambda|, & \text { if } 0<\lambda \lesssim 1, a+b+1=0 .
\end{array}
$$

Proof. See Lemma 4.2 in [14]. 
Lemma 3.3. Let $a>-1$ and $b>1$. For $\lambda_{1} \geq 0, \lambda_{2} \geq 1$ define

$$
F_{b}^{a}\left(\lambda_{1}, \lambda_{2}\right)=\int_{0}^{\lambda_{2}} \frac{\left(y+\lambda_{1}\right)^{a}}{(1+y)^{b}} d y
$$

Then,

1. If $\lambda_{1} \geq \lambda_{2}$,

$$
F_{b}^{a}\left(\lambda_{1}, \lambda_{2}\right) \approx \lambda_{1}^{a}
$$

2. If $0 \leq \lambda_{1} \leq 1 \leq \lambda_{2}$, then

$$
F_{b}^{a}\left(\lambda_{1}, \lambda_{2}\right) \approx \begin{cases}1+\log \lambda_{2}, & \text { if } \quad a-b+1=0 \\ \lambda_{2}^{\max \{a-b+1,0\}}, & \text { if } \quad a-b+1 \neq 0\end{cases}
$$

3. If $1 \leq \lambda_{1} \leq \lambda_{2}$, then

$$
\begin{aligned}
& F_{b}^{a}\left(\lambda_{1}, \lambda_{2}\right) \lesssim \lambda_{1}^{a}+\lambda_{2}^{a-b+1}, \text { if } a-b+1>0, \\
& F_{b}^{a}\left(\lambda_{1}, \lambda_{2}\right) \approx \lambda_{1}^{a}+\log \frac{\lambda_{2}}{\lambda_{1}}, \quad \text { if } a-b+1=0, \\
& F_{b}^{a}\left(\lambda_{1}, \lambda_{2}\right) \approx \lambda_{1}^{a}, \quad \text { if } a-b+1<0 .
\end{aligned}
$$

Proof. First assume that $\lambda_{1} \geq \lambda_{2}$. Then $y+\lambda_{1} \approx \lambda_{1}$ therefore,

$$
F_{b}^{a}\left(\lambda_{1}, \lambda_{2}\right)=\int_{0}^{\lambda_{2}} \frac{\left(y+\lambda_{1}\right)^{a}}{(1+y)^{b}} d y \approx \lambda_{1}^{a} \int_{0}^{\lambda_{2}} \frac{d y}{(1+y)^{b}} \approx \lambda_{1}^{a}
$$

Next, assume that $0 \leq \lambda_{1} \leq 1 \leq \lambda_{2}$. Split $F_{b}^{a}$ as follows:

$$
F_{b}^{a}\left(\lambda_{1}, \lambda_{2}\right)=\int_{0}^{1} \frac{\left(y+\lambda_{1}\right)^{a}}{(1+y)^{b}} d y+\int_{1}^{\lambda_{2}} \frac{\left(y+\lambda_{1}\right)^{a}}{(1+y)^{b}} d y
$$

The first integral is clearly $\approx 1$. For the second integral we have $y+\lambda_{1} \approx y$ and $1+y \approx y$, therefore,

$$
\int_{1}^{\lambda_{2}} \frac{\left(y+\lambda_{1}\right)^{a}}{(1+y)^{b}} d y \approx \int_{1}^{\lambda_{2}} y^{a-b} d y \approx\left\{\begin{array}{lll}
\log \lambda_{2}, & \text { if } & a-b+1=0 \\
\lambda_{2}^{\max \{a-b+1,0\}}, & \text { if } & a-b+1 \neq 0 .
\end{array}\right.
$$

Finally, assume that $1 \leq \lambda_{1} \leq \lambda_{2}$. Split $F_{b}^{a}$ as follows:

$$
F_{b}^{a}\left(\lambda_{1}, \lambda_{2}\right)=\int_{0}^{\lambda_{1}} \frac{\left(y+\lambda_{1}\right)^{a}}{(1+y)^{b}} d y+\int_{\lambda_{1}}^{\lambda_{2}} \frac{\left(y+\lambda_{1}\right)^{a}}{(1+y)^{b}} d y:=A+B
$$


In $A, y+\lambda_{1} \approx \lambda_{1}$ therefore,

$$
A \approx \lambda_{1}^{a} \int_{0}^{\lambda_{1}} \frac{1}{(1+y)^{b}} d y \approx \lambda_{1}^{a}
$$

In $B, y+\lambda_{1} \approx y$ and $1+y \approx y$, therefore

$$
\begin{aligned}
B & \approx \int_{\lambda_{1}}^{\lambda_{2}} y^{a-b} d y \\
& \approx \begin{cases}\lambda_{2}^{a-b+1}-\lambda_{1}^{a-b+1} \leq \lambda_{2}^{a-b+1}, & \text { if } a-b+1>0 \\
\log \frac{\lambda_{2}}{\lambda_{1}}, & \text { if } a-b+1=0 \\
\lambda_{1}^{a-b+1}-\lambda_{2}^{a-b+1} \leq \lambda_{1}^{a-b+1}=\lambda_{1}^{a} \lambda_{1}^{-(b-1)} \leq \lambda_{1}^{a}, & \text { if } a-b+1<0\end{cases}
\end{aligned}
$$

Lemma 3.4. Let $a>-1, b>1$. For $\lambda \geq 1$, define $G_{b}^{a}(\lambda)=\int_{0}^{\lambda} \frac{(\lambda-y)^{a}}{(1+y)^{b}} d y$. Then $G_{b}^{a}(\lambda) \approx \lambda^{a}$.

Proof.

$$
\begin{aligned}
G_{b}^{a}(\lambda) & =\int_{0}^{\frac{\lambda}{2}} \frac{(\lambda-y)^{a}}{(1+y)^{b}} d y+\int_{\frac{\lambda}{2}}^{\lambda} \frac{(\lambda-y)^{a}}{(1+y)^{b}} d y \\
& \approx \lambda^{a} \int_{0}^{\frac{\lambda}{2}} \frac{1}{(1+y)^{b}} d y+\frac{1}{\lambda^{b}} \int_{\frac{\lambda}{2}}^{\lambda}(\lambda-y)^{a} d y \approx \lambda^{a}+\lambda^{a-b+1} \approx \lambda^{a}
\end{aligned}
$$

Proof of Proposition 3.1. We may assume that $\tau>0$ because $J_{l}^{m}(-\tau, \xi)$ $=J_{l}^{m}(\tau, \xi)$ (to see this change variables $\theta \rightarrow \pi-\theta$ ). When $\tau+|\xi| \lesssim 1$ estimates (3.2) are easily seen to be true as $J_{l}^{m}(\tau, \xi) \lesssim 1$ and all the right-hand sides are then $\approx 1$. From now on we assume that $\tau+|\xi| \gtrsim 1$. Performing the change of variables $\theta \rightarrow x=\cos \theta$, we have

$$
J_{l}^{m}(\tau, \xi)=\int_{-1}^{1} \frac{\left(1-x^{2}\right)^{\frac{m-1}{2}}}{\left[1+(\tau+|\xi| x)^{2}\right]^{l}} d x .
$$

Write $J_{l}^{m}(\tau, \xi)=J_{+}+J_{-}$where

$$
\begin{aligned}
J_{+} & =\int_{0}^{1} \frac{\left(1-x^{2}\right)^{\frac{m-1}{2}}}{\left[1+(\tau+|\xi| x)^{2}\right]^{l}} d x \\
J_{-} & =\int_{-1}^{0} \frac{\left(1-x^{2}\right)^{\frac{m-1}{2}}}{\left[1+(\tau+|\xi| x)^{2}\right]^{l}} d x \\
& =\int_{0}^{1} \frac{\left(1-x^{2}\right)^{\frac{m-1}{2}}}{\left[1+(\tau-|\xi| x)^{2}\right]^{l}} d x
\end{aligned}
$$

Observe that $J_{+} \leq J_{-}$, hence $J_{l}^{m}(\tau, \xi) \approx J_{-}$, so it suffices to estimate $J_{-}$. We split the proof into two cases depending on the region where the pair $(\tau, \xi)$ lies. 
Case 1: Estimates inside the cone: Fix $(\tau, \xi)$ such that $\tau \geq|\xi|$. If $\tau \geq 2|\xi|$ use the fact that for $0 \leq x \leq 1,1+(\tau-|\xi| x)^{2} \approx 1+\tau^{2} \approx \tau^{2}$ to get,

$$
\begin{aligned}
J_{-} & =\int_{0}^{1} \frac{\left(1-x^{2}\right)^{\frac{m-1}{2}}}{\left[1+(\tau-|\xi| x)^{2}\right]^{l}} d x \\
& \approx \frac{1}{\tau^{2 l}} \int_{0}^{1}\left(1-x^{2}\right)^{\frac{m-1}{2}} d x \\
& \approx \frac{1}{\tau^{2 l}} \approx \frac{(1+\tau-|\xi|)^{\alpha}}{(1+\tau+|\xi|)^{2 l+\alpha}}
\end{aligned}
$$

It remains to deal with the case $|\xi|<\tau<2|\xi|$. Recall that we are assuming $\tau+|\xi| \gtrsim 1$, hence $|\xi| \gtrsim 1$. Using $1-x^{2} \approx 1-x$ and then changing variables $x \rightarrow y=1-x$ we have:

$$
\begin{aligned}
J_{-} & =\int_{0}^{1} \frac{y^{\frac{m-1}{2}}}{\left[1+(\tau-|\xi|+|\xi| y)^{2}\right]^{l}} d y \\
& \approx \int_{0}^{1} \frac{y^{\frac{m-1}{2}}}{[1+\tau-|\xi|+|\xi| y]^{2 l}} d y \\
& =\frac{1}{|\xi|^{2 l}} \int_{0}^{1} \frac{y^{\frac{m-1}{2}}}{\left[\frac{1+\tau-|\xi|}{|\xi|}+y\right]^{2 l}} d y \\
& =\frac{1}{|\xi|^{2 l}} H_{\frac{m-1}{2}}^{-2 l}\left(\frac{1+\tau-|\xi|}{|\xi|}\right)
\end{aligned}
$$

where $H_{\frac{m-1}{2}}^{-2 l}$ is defined in (3.3). Notice that $\frac{1+\tau-|\xi|}{|\xi|} \leq \frac{1+|\xi|}{|\xi|} \lesssim 1$. If $m+1-4 l \neq$ 0 then, by $(3.4 \mathrm{~b})$,

$$
H_{\frac{m-1}{2}}^{-2 l}\left(\frac{1+\tau-|\xi|}{|\xi|}\right) \approx\left(\frac{1+\tau-|\xi|}{|\xi|}\right)^{\min \left\{\frac{m+1}{2}-2 l, 0\right\}}=\left(\frac{1+\tau-|\xi|}{|\xi|}\right)^{\alpha}
$$

which gives,

$$
J_{-} \approx \frac{(1+\tau-|\xi|)^{\alpha}}{|\xi|^{2 l+\alpha}} \approx \frac{(1+\tau-|\xi|)^{\alpha}}{(1+\tau+|\xi|)^{2 l+\alpha}}
$$

If $m+1-4 l=0$ then, by $(3.4 \mathrm{c})$,

$$
H_{\frac{m-1}{2}}^{-2 l}\left(\frac{1+\tau-|\xi|}{|\xi|}\right) \approx 1+\left|\log \frac{1+\tau-|\xi|}{|\xi|}\right|
$$


which gives,

$$
\begin{aligned}
J_{-} & \approx \frac{1}{|\xi|^{2 l}}\left[1+\left|\log \frac{1+\tau-|\xi|}{|\xi|}\right|\right] \\
& \approx \frac{1}{(1+\tau+|\xi|)^{2 l}}\left[1+\log \frac{1+\tau+|\xi|}{1+\tau-|\xi|}\right]
\end{aligned}
$$

Case 2: Estimates outside the cone: Fix $(\tau, \xi)$ such that $0<\tau<|\xi|$. Recall that we are assuming $|\tau|+|\xi| \gtrsim 1$, hence $|\xi| \gtrsim 1$. By an appropriate choise of the constants we may assume that $|\xi| \geq 4$.

Suppose first that $|\xi| \geq 2 \tau$. Write $J_{-}=J_{-}^{(1)}+J_{-}^{(2)}$ where

$$
J_{-}^{(1)}=\int_{0}^{3 / 4} \frac{\left(1-x^{2}\right)^{\frac{m-1}{2}} d x}{\left[1+(\tau-|\xi| x)^{2}\right]^{l}}, \quad J_{-}^{(2)}=\int_{3 / 4}^{1} \frac{\left(1-x^{2}\right)^{\frac{m-1}{2}} d x}{\left[1+(\tau-|\xi| x)^{2}\right]^{l}}
$$

For $J_{-}^{(1)}$ use $1-x^{2} \approx 1$ and then change variables $x \rightarrow y=\tau-|\xi| x$ to get

$$
J_{-}^{(1)} \approx \frac{1}{|\xi|} \int_{-\left(\frac{3|\xi|}{4}-\tau\right)}^{\tau} \frac{d y}{\left[1+y^{2}\right]^{l}} \leq \frac{1}{|\xi|} \int_{-\infty}^{+\infty} \frac{d y}{\left[1+y^{2}\right]^{l}} \approx \frac{1}{|\xi|}
$$

Since $\frac{3|\xi|}{4}-\tau \geq \frac{|\xi|}{4} \geq 1$, we also have $\int_{-\left(\frac{3|\xi|}{4}-\tau\right)}^{\tau} \frac{d y}{\left[1+y^{2}\right]^{l}} \geq \int_{-1}^{0} \frac{d y}{\left[1+y^{2}\right]^{l}} \gtrsim 1$, therefore $J_{-}^{(1)} \approx \frac{1}{|\xi|}$. For $J_{-}^{(2)}$ use $|\xi| x-\tau \approx|\xi|$, to get:

$$
J_{-}^{(2)} \approx \frac{1}{|\xi|^{2 l}} \int_{3 / 4}^{1}(1-x)^{\frac{m-1}{2}} d x \lesssim \frac{1}{|\xi|}
$$

Therefore $J_{-} \approx \frac{1}{|\xi|}\left({ }^{5}\right)$.

Suppose next that $\tau<|\xi|<2 \tau$ and $|\xi|-\tau \leq 1$. Changing variables $x \rightarrow y=|\xi|(1-x)$ we get:

$$
J_{-} \approx \int_{0}^{1} \frac{(1-x)^{\frac{m-1}{2}} d x}{\left[1+(\tau-|\xi| x)^{2}\right]^{l}}=\frac{1}{|\xi|^{\frac{m+1}{2}}} \int_{0}^{|\xi|} \frac{y^{\frac{m-1}{2}} d y}{\left[1+(y-(|\xi|-\tau))^{2}\right]^{l}}
$$

Observe that, since $0 \leq|\xi|-\tau \leq 1$, we have $1+(y-(|\xi|-\tau))^{2} \approx 1+y^{2}$ (if $y \lesssim 1$ then both sides are $\approx 1$, and if $y \gtrsim 1$ then both sides are $\approx y^{2}$ ),

\footnotetext{
${ }^{5}$ Note that the decay rate is independent of the parameters $m$ and $l$. This estimate shows that, in the various versions of the averaging Lemmas, we cannot expect a gain better than $1 / 2$ derivatives. Also note that this 'worse decay' occurs when $|\xi|>>\tau$.
} 
therefore, using (3.7), we have

$$
\begin{aligned}
& J_{-} \approx \frac{1}{|\xi|^{\frac{m+1}{2}}} \int_{0}^{|\xi|} \frac{y^{\frac{m-1}{2}} d y}{\left[1+y^{2}\right]^{l}} \approx \frac{1}{|\xi|^{\frac{m+1}{2}}} F_{2 l}^{\frac{m-1}{2}}(0,|\xi|) \\
& \approx \begin{cases}\frac{1}{\left.|\xi|^{\min \left\{\frac{m+1}{2}, 2 l\right.}\right\}}, & \text { if } m+1-4 l \neq 0 \\
\frac{1}{|\xi|^{2 l}[1+\log |\xi|],}, & \text { if } m+1-4 l=0\end{cases} \\
& \approx \begin{cases}\frac{(1+|\xi|-\tau)^{2 l-1+\alpha}}{(1+|\xi|+\tau)^{2 l+\alpha}}, & \text { if } m+1-4 l \neq 0 \\
\frac{(1+|\xi|-\tau)^{2 l-1}}{(1+|\xi|+\tau)^{2 l}}\left[1+\log \frac{1+|\xi|+\tau}{1+|\xi|-\tau}\right], & \text { if } m+1-4 l=0\end{cases}
\end{aligned}
$$

It remains to deal with the case $\tau<|\xi|<2 \tau$ and $|\xi|-\tau>1$. Change variables $x \rightarrow y=\tau-|\xi| x$ and then split $J_{-}$as follows:

$$
\begin{aligned}
J_{-} & \approx \int_{0}^{1} \frac{(1-x)^{\frac{m-1}{2}} d x}{\left[1+(\tau-|\xi| x)^{2}\right]^{l}}=\frac{1}{|\xi|^{\frac{m+1}{2}}} \int_{-(|\xi|-\tau)}^{\tau} \frac{(y+|\xi|-\tau)^{\frac{m-1}{2}} d y}{\left[1+y^{2}\right]^{l}} \\
& =\frac{1}{|\xi|^{\frac{m+1}{2}}} \int_{0}^{\tau} \frac{(y+|\xi|-\tau)^{\frac{m-1}{2}} d y}{\left[1+y^{2}\right]^{l}}+\frac{1}{|\xi|^{\frac{m+1}{2}}} \int_{0}^{|\xi|-\tau} \frac{(-y+|\xi|-\tau)^{\frac{m-1}{2}} d y}{\left[1+y^{2}\right]^{l}} \\
& \approx \frac{1}{|\xi|^{\frac{m+1}{2}}} F_{2 l}^{\frac{m-1}{2}}(|\xi|-\tau, \tau)+\frac{1}{|\xi|^{\frac{m+1}{2}}} G_{2 l}^{\frac{m-1}{2}}(|\xi|-\tau) \\
& \approx \frac{1}{|\xi|^{\frac{m+1}{2}}} F_{2 l}^{\frac{m-1}{2}}(|\xi|-\tau, \tau)+\frac{(|\xi|-\tau)^{\frac{m-1}{2}}}{|\xi|^{\frac{m+1}{2}}}
\end{aligned}
$$

where we have used Lemma 3.4 in the last step. If $m+1-4 l<0$ then, using (3.8c) of Lemma 3.3, we obtain:

$$
J_{-} \approx \frac{(|\xi|-\tau)^{\frac{m-1}{2}}}{|\xi|^{\frac{m+1}{2}}} \approx \frac{(1+|\xi|-\tau)^{2 l-1+\alpha}}{(1+|\xi|+\tau)^{2 l+\alpha}}
$$

If $m+1-4 l=0$ (hence $\left.\frac{m-1}{2}=2 l-1>0\right)$ then, using (3.8b) of Lemma 3.3, we obtain:

$$
\begin{aligned}
J_{-} & \approx \frac{(|\xi|-\tau)^{\frac{m-1}{2}}}{|\xi|^{\frac{m+1}{2}}}+\frac{1}{|\xi|^{\frac{m+1}{2}}} \log \frac{\tau}{|\xi|-\tau} \\
& \lesssim \frac{(|\xi|-\tau)^{\frac{m-1}{2}}}{|\xi|^{\frac{m+1}{2}}}\left[1+\log \frac{\tau}{|\xi|-\tau}\right] \\
& \approx \frac{(1+|\xi|-\tau)^{2 l-1}}{(1+|\xi|+\tau)^{2 l}}\left[1+\log \frac{1+|\xi|+\tau}{1+|\xi|-\tau}\right]
\end{aligned}
$$


If $m+1-4 l>0$ then, using (3.8a) of Lemma 3.3, we obtain:

$$
\begin{aligned}
J_{-} & \approx \frac{(|\xi|-\tau)^{\frac{m-1}{2}}}{|\xi|^{\frac{m+1}{2}}}+\frac{\tau^{\frac{m+1}{2}-2 l}}{|\xi|^{\frac{m+1}{2}}} \\
& \approx \frac{(|\xi|-\tau)^{2 l-1}}{|\xi|^{2 l}}\left(\frac{|\xi|-\tau}{|\xi|}\right)^{\frac{m+1}{2}-2 l}+\frac{1}{|\xi|^{2 l}} \lesssim \frac{(|\xi|-\tau)^{2 l-1}}{|\xi|^{2 l}} \\
& \approx \frac{(1+|\xi|-\tau)^{2 l-1+\alpha}}{(1+|\xi|+\tau)^{2 l+\alpha}} \quad(\alpha=0 \text { in this case })
\end{aligned}
$$

Remark 3.5. Our proof shows that estimate (3.2b) is sharp in all cases except when $m+1-4 l \geq 0$ and $(\tau, \xi)$ is in the region $|\xi|-|\tau|>1$ and $|\tau|<|\xi|<2|\tau|$. In this case the sharp estimate is (see (3.20) and (3.22)):

$$
\begin{array}{ll}
J_{l}^{m}(\tau, \xi) \approx \frac{(|\xi|-|\tau|)^{\frac{m-1}{2}}}{|\xi|^{\frac{m+1}{2}}}+\frac{|\tau|^{\frac{m+1}{2}-2 l}}{|\xi|^{\frac{m+1}{2}}}, & \text { if } m+1-4 l>0 \\
J_{l}^{m}(\tau, \xi) \approx \frac{(|\xi|-|\tau|)^{\frac{m-1}{2}}}{|\xi|^{\frac{m+1}{2}}}+\frac{1}{|\xi|^{\frac{m+1}{2}}} \log \frac{|\tau|}{|\xi|-|\tau|}, \text { if } m+1-4 l=0
\end{array}
$$

In particular in the case $|\tau| \leq|\xi|$ and $m+1-4 l<0(3.2 \mathrm{~b})$ is sharp and gives:

$$
J_{l}^{m}(\tau, \xi) \approx \frac{(1+|\xi|-|\tau|)^{\frac{m-1}{2}}}{(1+|\xi|+|\tau| \mid)^{\frac{m+1}{2}}} .
$$

Proof of Theorem 2. By taking the Fourier transform with respect to the spacetime variables in (1.1) it is easy to see that

$$
\tilde{\rho}(\tau, \xi)=\int_{\mathbb{S}^{d-1}} \tilde{f}(\tau, \xi, v) d \sigma(v)=\int_{\mathbb{S}^{d-1}} \frac{\tilde{h}(\tau, \xi, v)}{1+i(\tau+v \cdot \xi)} d \sigma(v) .
$$

where $h=f+g$. Apply the Cauchy-Schwarz inequality to get:

$$
|\tilde{\rho}(\tau, \xi)|^{2} \leq\left(\int_{\mathbb{S}^{d-1}} \frac{d \sigma(v)}{1+(\tau+v \cdot \xi)^{2}}\right)\left(\int_{\mathbb{S}^{d-1}}|\tilde{h}(\tau, \xi, v)|^{2} d \sigma(v)\right) .
$$

We can use a basis in $v$-space such that $\xi=(0, \ldots, 0,|\xi|)$. Thus, using spherical coordinates we get that

$$
\int_{\mathbb{S}^{d-1}} \frac{d \sigma(v)}{1+(\tau+v \cdot \xi)^{2}} \lesssim \int_{0}^{\pi} \frac{(\sin \theta)^{d-2}}{1+(\tau+|\xi| \cos \theta)^{2}} d \theta=J_{1}^{d-2}(\tau, \xi)
$$

The estimates in Theorem 2 are proved by first applying the pointwise estimates for $J_{1}^{d-2}(\tau, \xi)$ given in Proposition 3.1, and then integrating with respect to $(\tau, \xi)$. 
Proof of Theorem 1. We present the proof in the case $d \neq 5$. The case $d=5$ is similar. Fix $(s, \delta)$ with $s+\delta \leq \frac{1}{2}$ and $s \leq 1+\mathrm{m}$, where $\mathrm{m}=\min \{(d-5) / 4,0\}$. Set $w_{+}(\tau, \xi)=1+|\tau|+|\xi|$ and $w_{-}=1+|| \tau|-| \xi||$. Theorem 2 implies that

$$
\left\|w_{+}^{1+\mathrm{m}} w_{-}^{-\frac{1}{2}-\mathrm{m}} \tilde{\rho}\right\|_{L^{2}\left(\mathbb{R}^{1+d}\right)} \lesssim\|f\|_{L^{2}\left(\mathbb{R}^{1+d} \times \mathbb{S}^{d-1}\right)}+\|g\|_{L^{2}\left(\mathbb{R}^{1+d} \times \mathbb{S}^{d-1}\right)}
$$

so it suffices to show that

$$
w_{+}^{s} w_{-}^{\delta} \leq w_{+}^{1+\mathrm{m}} w_{-}^{-\frac{1}{2}-\mathrm{m}}
$$

Indeed,

$$
\begin{aligned}
w_{+}^{s} w_{-}^{\delta} & \leq w_{+}^{s} w_{-}^{\frac{1}{2}-s} \quad\left(\text { because } \delta \leq \frac{1}{2}-s\right) \\
& =w_{+}^{1+\mathrm{m}} w_{-}^{-\frac{1}{2}-\mathrm{m}}\left(\frac{w_{-}}{w_{+}}\right)^{1+\mathrm{m}-s} \\
& \leq w_{+}^{1+\mathrm{m}} w_{-}^{-\frac{1}{2}-\mathrm{m}} \quad(\text { because } 1+\mathrm{m}-s \geq 0)
\end{aligned}
$$

\section{Averaging Lemmas for $\partial_{t} f+v \cdot \nabla_{x} f=\Omega_{v}^{i, j} g$ in $2+1$ dimensions}

Proof of Theorem 3. Since $d=2$, we can set $(i, j)=(1,2)$. Take the spacetime Fourier transform in (1.18) and add $\lambda \tilde{f}(\tau, \xi, v)$ to both sides to obtain that

$$
(\lambda+i(\tau+v \cdot \xi)) \tilde{f}(\tau, \xi, v)=\Omega_{v}^{1,2} \tilde{g}(\tau, \xi, v)+\lambda \tilde{f}(\tau, \xi, v)
$$

The parameter $\lambda=\lambda(\tau, \xi)$ depends on $(\tau, \xi)$ and will be chosen later. Then,

$$
\tilde{\rho}(\tau, \xi)=\int_{\mathbb{S}^{1}} \frac{\Omega_{v}^{1,2} \tilde{g}(\tau, \xi, v)}{\lambda+i(\tau+v \cdot \xi)} d \sigma(v)+\int_{\mathbb{S}^{1}} \frac{\lambda \tilde{f}(\tau, \xi, v)}{\lambda+i(\tau+v \cdot \xi)} d \sigma(v) .
$$

Integrate by parts in the first term to get:

$$
\tilde{\rho}(\tau, \xi)=-i \int_{\mathbb{S}^{1}} \tilde{g}(\tau, \xi, v) \frac{v_{1} \xi_{2}-v_{2} \xi_{1}}{(\lambda+i(\tau+v \cdot \xi))^{2}} d \sigma(v)+\int_{\mathbb{S}^{1}} \frac{\lambda \tilde{f}(\tau, \xi, v) d \sigma(v)}{\lambda+i(\tau+v \cdot \xi)} .
$$

Therefore,

$$
|\tilde{\rho}(\tau, \xi)|^{2} \leq\|\tilde{g}(\tau, \xi, v)\|_{L_{v}^{2}}^{2} I_{1}(\tau, \xi)+\|\tilde{f}(\tau, \xi, v)\|_{L_{v}^{2}}^{2} I_{2}(\tau, \xi),
$$

where

$$
I_{1}(\tau, \xi)=\int_{\mathbb{S}^{1}} \frac{\left|v_{1} \xi_{2}-v_{2} \xi_{1}\right|^{2}}{\left(\lambda^{2}+(\tau+\xi \cdot v)^{2}\right)^{2}} d \sigma(v)
$$


and

$$
I_{2}(\tau, \xi)=\lambda^{2} \int_{\mathbb{S}^{1}} \frac{d \sigma(v)}{\lambda^{2}+(\tau+\xi \cdot v)^{2}} .
$$

Set $\tau^{\prime}=\frac{\tau}{\lambda}$ and $\xi^{\prime}=\frac{\xi}{\lambda}$. Then

$$
\begin{aligned}
I_{1}(\tau, \xi) & =\frac{|\xi|^{2}}{\lambda^{4}} \int_{\mathbb{S}^{1}} \frac{\left|v_{1} \frac{\xi_{2}}{|\xi|}-v_{2} \frac{\xi_{1}}{|\xi|}\right|^{2}}{\left(1+\left(\tau^{\prime}+\xi^{\prime} \cdot v\right)^{2}\right)^{2}} d \sigma(v) \\
& \lesssim \frac{|\xi|^{2}}{\lambda^{4}} \int_{0}^{\pi} \frac{(\sin \theta)^{2}}{\left[1+\left(\tau^{\prime}+\left|\xi^{\prime}\right| \cos \theta\right)^{2}\right]^{2}} d \theta \\
& =\frac{|\xi|^{2}}{\lambda^{4}} J_{2}^{2}\left(\tau^{\prime}, \xi^{\prime}\right)
\end{aligned}
$$

and

$$
I_{2}(\tau, \xi)=\int_{\mathbb{S}^{1}} \frac{d \sigma(v)}{1+\left(\tau^{\prime}+\xi^{\prime} \cdot v\right)^{2}} \lesssim \int_{0}^{\pi} \frac{d \theta}{1+\left(\tau^{\prime}+\left|\xi^{\prime}\right| \cos \theta\right)^{2}}=J_{1}^{0}(\tau, \xi)
$$

with the $J_{l}^{m}$-integrals defined as in (3.1). As a consequence of (3.2) we have, in all regions,

$$
J_{l}^{m}\left(\tau^{\prime}, \xi^{\prime}\right) \lesssim \frac{\left(1+|| \xi^{\prime}|-| \tau^{\prime}||\right)^{2 l-1+\alpha}}{\left(1+\left|\xi^{\prime}\right|+\left|\tau^{\prime}\right|\right)^{2 l+\alpha}}
$$

provided that $\alpha=\min \left\{\frac{m+1-4 l}{2}, 0\right\} \neq 0$.

With $(\tau, \xi)$ fixed, choose $\lambda=\lambda(\tau, \xi)$ such that

$$
\lambda>1 \text { and } \lambda=(\lambda+|\xi|+|\tau|)^{\frac{1}{4}}(\lambda+|| \xi|-| \tau||)^{\frac{1}{4}}
$$

Then

$$
\begin{aligned}
I_{1}(\tau, \xi) & \lesssim \frac{|\xi|^{2}}{\lambda^{4}} J_{2}^{2}\left(\tau^{\prime}, \xi^{\prime}\right) \\
& \lesssim \frac{|\xi|^{2}}{\lambda^{4}} \frac{\left(1+|| \xi^{\prime}|-| \tau^{\prime}||\right)^{\frac{1}{2}}}{\left(1+\left|\xi^{\prime}\right|+\left|\tau^{\prime}\right|\right)^{\frac{3}{2}}} \\
& \leq \frac{(\lambda+|\xi|+|\tau|)^{2}}{\lambda^{4}} \frac{\lambda(\lambda+|| \xi|-| \tau||)^{\frac{1}{2}}}{(\lambda+|\xi|+|\tau|)^{\frac{3}{2}}} \\
& =\frac{(\lambda+|\xi|+|\tau|)^{\frac{1}{2}}(\lambda+|| \xi|-| \tau||)^{\frac{1}{2}}}{\lambda^{3}} \\
& =\frac{1}{(\lambda+|\xi|+|\tau|)^{\frac{1}{4}}(\lambda+|| \xi|-| \tau||)^{\frac{1}{4}}} \\
& \leq \frac{1}{(1+|\xi|+|\tau|)^{\frac{1}{4}}(1+|| \xi|-| \tau||)^{\frac{1}{4}}}
\end{aligned}
$$


and

$$
\begin{aligned}
& I_{2}(\tau, \xi) \lesssim J_{1}^{0}\left(\tau^{\prime}, \xi^{\prime}\right) \lesssim \frac{1}{\left(1+\left|\xi^{\prime}\right|+\left|\tau^{\prime}\right|\right)^{\frac{1}{2}}\left(1+|| \xi^{\prime}|-| \tau^{\prime}||\right)^{\frac{1}{2}}} \\
& =\frac{\lambda}{(\lambda+|\xi|+|\tau|)^{\frac{1}{2}}(\lambda+|| \xi|-| \tau||)^{\frac{1}{2}}}=\frac{1}{(\lambda+|\xi|+|\tau|)^{\frac{1}{4}}(\lambda+|| \xi|-| \tau||)^{\frac{1}{4}}}
\end{aligned}
$$

$$
\leq \frac{1}{(1+|\xi|+|\tau|)^{\frac{1}{4}}(1+|| \xi|-| \tau||)^{\frac{1}{4}}}
$$

Combining (4.1), (4.6) and (4.7) in the usual way we get (1.20). Next we prove (1.19). With $(\tau, \xi)$ fixed, choose a new $\lambda=\lambda(\tau, \xi)$ such that

$$
\lambda>1 \text { and } \lambda=(\lambda+|\xi|+|\tau|)^{\frac{1}{6}}(\lambda+|| \xi|-| \tau||)^{\frac{1}{2}}
$$

Then

$$
\begin{aligned}
I_{1}(\tau, \xi) & \lesssim \frac{|\xi|^{2}}{\lambda^{4}} J_{2}^{2}\left(\tau^{\prime}, \xi^{\prime}\right) \\
& \lesssim \frac{|\xi|^{2}}{\lambda^{4}} \frac{\left(1+|| \xi^{\prime}|-| \tau^{\prime}||\right)^{\frac{1}{2}}}{\left(1+\left|\xi^{\prime}\right|+\left|\tau^{\prime}\right|\right)^{\frac{3}{2}}} \\
& \lesssim \frac{|\xi|^{2}}{\lambda^{4}} \frac{\left(1+|| \xi^{\prime}|-| \tau^{\prime}||\right)^{\frac{5}{2}}}{\left(1+\left|\xi^{\prime}\right|+\left|\tau^{\prime}\right|\right)^{\frac{3}{2}}} \\
& \lesssim \frac{(\lambda+|\xi|+|\tau|)^{2}}{\lambda^{4}} \frac{(\lambda+|| \xi|-| \tau||)^{\frac{5}{2}}}{\lambda(\lambda+|\xi|+|\tau|)^{\frac{3}{2}}} \\
& =\frac{(\lambda+|\xi|+|\tau|)^{\frac{1}{2}}(\lambda+|| \xi|-| \tau||)^{\frac{5}{2}}}{\lambda^{5}} \\
& =\frac{1}{(\lambda+|\xi|+|\tau|)^{\frac{1}{3}}} \\
& \leq \frac{1}{(1+|\xi|+|\tau|)^{\frac{1}{3}}}
\end{aligned}
$$

and

$$
\begin{aligned}
I_{2}(\tau, \xi) & \lesssim J_{1}^{0}\left(\tau^{\prime}, \xi^{\prime}\right) \lesssim \frac{1}{\left(1+\left|\xi^{\prime}\right|+\left|\tau^{\prime}\right|\right)^{\frac{1}{2}}\left(1+|| \xi^{\prime}|-| \tau^{\prime}||\right)^{\frac{1}{2}}} \\
& =\frac{\lambda}{(\lambda+|\xi|+|\tau|)^{\frac{1}{2}}(\lambda+|| \xi|-| \tau||)^{\frac{1}{2}}}=\frac{1}{(\lambda+|\xi|+|\tau|)^{\frac{1}{3}}} \\
& \leq \frac{1}{(1+|\xi|+|\tau|)^{\frac{1}{3}}}
\end{aligned}
$$

Combining (4.1), (4.10) and (4.11) in the usual way we get (1.19). 
Remark 4.1. The estimates for the $J_{l}^{m}$ integrals are applied with $m=d-2$ (see for example (3.27) in last Section). In this sense $J_{2}^{2}$ corresponds to $d=4$ and behaves like a four dimensional term while $J_{1}^{0}$ corresponds to $d=2$ and it is a two dimensional term. It is the sharp estimates for these integrals in higher dimensions that allow us to improve the results of [6].

Corollary 4.2. Under the assumptions and notation of Theorem 3, the average over the sphere satisfies:

$$
\begin{aligned}
\left\|(1+|\tau|+|\xi|)^{s}(1+\| \tau|-| \xi||)^{\delta} \tilde{\rho}(\tau, \xi)\right\|_{L^{2}\left(\mathbb{R}^{1+2}\right)} & \\
& \lesssim\left(\|f\|_{L^{2}\left(\mathbb{R}^{1+2}, \mathbb{S}^{1}\right)}+\|g\|_{L^{2}\left(\mathbb{R}^{1+2}, \mathbb{S}^{1}\right)}\right)
\end{aligned}
$$

whenever the pair $(s, \delta)$ satisfies

$$
s+\delta \leq 1 / 4,6 s+2 \delta \leq 1, \text { and } s \leq 1 / 6
$$

Proof. Define $w_{+}=1+|\xi|+|\tau|$ and $w_{-}=1+|| \xi|-| \tau||$. Interpolating between the two estimates in Theorem 3 we easily get (4.12) for any pair $(s, \delta)$ lying on the line segment with endpoints $(s, \delta)=(1 / 6,0)$ and $(s, \delta)=$ $(1 / 8,1 / 8)$. Now fix $(s, \delta)$ as in (4.13). If $s \leq \frac{1}{8}$ then

$$
w_{+}^{s} w_{-}^{\delta} \leq w_{+}^{s} w_{-}^{\frac{1}{4}-s}=w_{+}^{\frac{1}{8}} w_{-}^{\frac{1}{8}}\left(\frac{w_{-}}{w_{+}}\right)^{\frac{1}{8}-s} \leq w_{+}^{\frac{1}{8}} w_{-}^{\frac{1}{8}}
$$

and the result follows from (1.20). If on the other hand $\frac{1}{8} \leq s \leq \frac{1}{6}$ then

$$
w_{+}^{s} w_{-}^{\delta} \leq w_{+}^{s} w_{-}^{\frac{1-6 s}{2}}
$$

and again the result follows as $\left(s, \frac{1-6 s}{2}\right)$ lies on the line segment with endpoints $(s, \delta)=(1 / 6,0)$ and $(s, \delta)=(1 / 8,1 / 8)$.

\section{Averaging Lemmas for the Initial Value Problem}

In this section, we continue the investigation started in [6] on studying the smoothing effect on the averages over the sphere:

$$
\rho(t, x)=\int_{\mathbb{S}^{d-1}} f(t, x, v) d \sigma(v),
$$

where $f: \mathbb{R}^{1+d} \times \mathbb{S}^{d-1} \rightarrow \mathbb{R}$ is a solution of the homogeneous initial value problem:

$$
\left\{\begin{array}{l}
\partial_{t} f+v \cdot \nabla_{x} f=0 \\
f(0, x, v)=f_{0}(x, v)
\end{array}\right.
$$


Proof of Theorem 4. We have

$$
\widetilde{\rho}(\tau, \xi)=\int_{\mathbb{S}^{d-1}} \hat{f}_{0}(\xi, v) \delta(\tau+\xi \cdot v) d \sigma(v) .
$$

and supp $\tilde{\rho} \subseteq\{(\tau, \xi):|\xi| \geq|\tau|\}$. We prove first the following two pointwise estimates:

$$
\begin{array}{r}
\int_{-|\xi|}^{|\xi|}|\xi|^{\frac{d-1}{2}}(|\xi|-|\tau|)^{-\frac{d-3}{2}}|\widetilde{\rho}(\tau, \xi)|^{2} d \tau \lesssim \int_{\mathbb{S}^{d-1}}\left|\hat{f}_{0}(\xi, v)\right|^{2} d \sigma(v) \\
|\tilde{\rho}(\tau, \xi)|^{2} \lesssim \frac{(|\xi|-|\tau|)^{\frac{d-3}{2}}}{|\xi|^{\frac{d-1}{2}}} \int_{\mathbb{S}^{d-1}}\left|\hat{f}_{0}(\xi, v)\right|^{2} d \sigma(v)
\end{array}
$$

Fix $(\tau, \xi)$ with $|\tau|<|\xi|$. Then

$$
\begin{gathered}
\widetilde{\rho}(\tau, \xi)=\int_{\mathbb{S}^{d-2}} \int_{0}^{\pi} \hat{f}_{0}(\xi, \omega \sin \theta, \cos \theta) \delta(\tau+|\xi| \cos \theta)(\sin \theta)^{d-2} d \theta d \sigma(\omega) \\
=\frac{1}{|\xi|} \int_{\mathbb{S}^{d-2}} \int_{\tau-|\xi|}^{\tau+|\xi|} \hat{f}_{0}\left(\xi, \omega \sqrt{1-\left(\frac{x-\tau}{|\xi|}\right)^{2}}, \frac{x-\tau}{|\xi|}\right) . \\
\cdot\left(1-\left(\frac{x-\tau}{|\xi|}\right)^{2}\right)^{\frac{d-3}{2}} \delta(x) d x d \sigma(\omega)
\end{gathered}
$$

$$
=\frac{1}{|\xi|} \int_{\mathbb{S}^{d-2}} \hat{f}_{0}\left(\xi, \omega \sqrt{1-\left(\frac{\tau}{|\xi|}\right)^{2}},-\frac{\tau}{|\xi|}\right)\left(1-\left(\frac{\tau}{|\xi|}\right)^{2}\right)^{\frac{d-3}{2}} d \sigma(\omega)
$$

where we have performed the change of variables $x=\tau+|\xi| \cos \theta$ in obtaining the second identity. Using a trivial Cauchy-Schwarz inequality on $\mathbb{S}^{d-2}$, we get that

$$
|\xi|^{2}|\widetilde{\rho}(\tau, \xi)|^{2} \lesssim \int_{\mathbb{S}^{d-2}}\left|\hat{f}_{0}\left(\xi, \omega \sqrt{1-\left(\frac{\tau}{|\xi|}\right)^{2}},-\frac{\tau}{|\xi|}\right)\right|^{2}\left(1-\left(\frac{\tau}{|\xi|}\right)^{2}\right)^{d-3} d \sigma(\omega),
$$

or, equivalently,

$$
\begin{aligned}
& \frac{|\xi|^{2}}{\left(1-\left(\frac{\tau}{|\xi|}\right)^{2}\right)^{\frac{d-3}{2}}}|\widetilde{\rho}(\tau, \xi)|^{2} \\
& \quad \lesssim \int_{\mathbb{S}^{d-2}}\left|\hat{f}_{0}\left(\xi, \omega \sqrt{1-\left(\frac{\tau}{|\xi|}\right)^{2}},-\frac{\tau}{|\xi|}\right)\right|^{2}\left(1-\left(\frac{\tau}{|\xi|}\right)^{2}\right)^{\frac{d-3}{2}} d \sigma(\omega) .
\end{aligned}
$$


Now integrate with respect to $\tau$ (recall that $|\tau|<|\xi|$ ) and then change variables $\tau \rightarrow \theta$ where $\cos \theta=-\tau /|\xi|$ to obtain:

$$
\begin{aligned}
& \int_{-|\xi|}^{|\xi|} \frac{|\xi|^{d-1}}{(|\xi|-|\tau|)^{\frac{d-3}{2}}(|\xi|+|\tau|)^{\frac{d-3}{2}}}|\widetilde{\rho}(\tau, \xi)|^{2} d \tau \\
& \quad \lesssim \int_{-|\xi|}^{|\xi|} \int_{\mathbb{S}^{d-2}}\left|\hat{f}_{0}\left(\xi, \omega \sqrt{1-\left(\frac{\tau}{|\xi|}\right)^{2}},-\frac{\tau}{|\xi|}\right)\right|^{2}\left(1-\left(\frac{\tau}{|\xi|}\right)^{2}\right)^{\frac{d-3}{2}} d \sigma(\omega) d \tau \\
& \quad=|\xi| \int_{0}^{\pi} \int_{\mathbb{S}^{d-2}}(\sin \theta)^{d-2}\left|\hat{f}_{0}(\xi, \omega \sin \theta, \cos \theta)\right|^{2} d \sigma(\omega) d \theta \\
& \quad=|\xi| \int_{\mathbb{S}^{d-1}}\left|\hat{f}_{0}(\xi, v)\right|^{2} d \sigma(v) .
\end{aligned}
$$

Using $|\tau|+|\xi| \approx|\xi|$ we obtain $(5.4)\left({ }^{6}\right)$.

Going back to (5.3) and applying Cauchy-Schwarz we get

$$
|\tilde{\rho}(\tau, \xi)|^{2} \leq I(\tau, \xi) \int_{\mathbb{S}^{d-1}}\left|\hat{f}_{0}(\xi, v)\right|^{2} d \sigma(v)
$$

where

$$
I(\tau, \xi)=\int_{\mathbb{S}^{d-1}} \delta(\tau+v \cdot \xi) d \sigma(v)=C \int_{0}^{\pi} \delta(\tau+|\xi| \cos \theta)(\sin \theta)^{d-2} d \theta
$$

We change variables $\theta \rightarrow x=\tau+|\xi| \cos \theta$ and use $|\xi|+|\tau| \approx|\xi|$ to get:

$$
\begin{aligned}
I(\tau, \xi) & =\int_{-(|\xi|-\tau)}^{|\xi|+\tau}\left(1-\left(\frac{x-\tau}{|\xi|}\right)^{2}\right)^{\frac{d-3}{2}} \frac{\delta(x) d x}{|\xi|} \\
& =\frac{1}{|\xi|}\left(1-\left(\frac{\tau}{|\xi|}\right)^{2}\right)^{\frac{d-3}{2}} \\
& =\frac{(|\xi|-|\tau|)^{\frac{d-3}{2}}}{|\xi|^{\frac{d-1}{2}}}
\end{aligned}
$$

This proves (5.5). Now we write

$$
\left\|(1+|\tau|+|\xi|)^{\frac{d-1}{4}}(1+\| \tau|-| \xi||)^{-\frac{d-3}{4}} \widetilde{\rho}(\tau, \xi)\right\|_{L^{2}\left(\mathbb{R}^{1+d}\right)}^{2}=A+B
$$

\footnotetext{
${ }^{6}$ Strictly speaking our proof of (5.4) makes sense only for $d \geq 3$, but the case $d=2$ requires only minor modifications of the argument. See Section 5 of [6] for the proof in two dimensions.
} 
where

$$
\begin{aligned}
& A=\int_{\mathbb{R}^{d}} \int_{0 \leq|\xi|-|\tau| \leq 1}(1+|\tau|+|\xi|)^{\frac{d-1}{2}}(1+|\xi|-|\tau|)^{-\frac{d-3}{2}}|\widetilde{\rho}(\tau, \xi)|^{2} d \tau d \xi \\
& B=\int_{\mathbb{R}^{d}} \int_{|\xi|-|\tau| \geq 1}(1+|\tau|+|\xi|)^{\frac{d-1}{2}}(1+|\xi|-|\tau|)^{-\frac{d-3}{2}}|\widetilde{\rho}(\tau, \xi)|^{2} d \tau d \xi
\end{aligned}
$$

To estimate $A$ we first use $1+|\tau|+|\xi| \approx 1+|\xi|$ and $1+|\xi|-|\tau| \approx 1$ and then apply estimate (5.5) to get:

$$
A \lesssim \int_{\mathbb{R}^{d}} \int_{\mathbb{S}^{d-1}}\left|\widehat{f}_{0}(\xi, v)\right|^{2}\left[\frac{(1+|\xi|)^{\frac{d-1}{2}}}{|\xi|^{\frac{d-1}{2}}} \int_{0 \leq|\xi|-|\tau| \leq 1}(|\xi|-|\tau|)^{\frac{d-3}{2}} d \tau\right] d \sigma(v) d \xi
$$

If $|\xi| \geq 1$ then

$$
\frac{(1+|\xi|)^{\frac{d-1}{2}}}{|\xi|^{\frac{d-1}{2}}} \int_{0 \leq|\xi|-|\tau| \leq 1}(|\xi|-|\tau|)^{\frac{d-3}{2}} d \tau \lesssim \int_{|\xi|-1}^{|\xi|}(|\xi|-x)^{\frac{d-3}{2}} d x \lesssim 1
$$

and if $|\xi| \leq 1$ then

$$
\frac{(1+|\xi|)^{\frac{d-1}{2}}}{|\xi|^{\frac{d-1}{2}}} \int_{0 \leq|\xi|-|\tau| \leq 1}(|\xi|-|\tau|)^{\frac{d-3}{2}} d \tau \lesssim \frac{1}{|\xi|^{\frac{d-1}{2}}} \int_{0}^{|\xi|}(|\xi|-x)^{\frac{d-3}{2}} d x \lesssim 1
$$

therefore

$$
A \lesssim\left\|f_{0}\right\|_{L^{2}\left(\mathbb{R}^{d} \times \mathbb{S}^{d-1}\right)}^{2}
$$

For $B$ we have $1+|\tau|+|\xi| \approx|\xi|$ and $1+|\xi|-|\tau| \approx|\xi|-|\tau|$, therefore the corresponding estimate follows by integrating (5.4) w.r.t $\xi$.

Remark 5.1. Integrating (5.5) w.r.t. $\xi$ we obtain that the following estimate is also true:

$$
\left.\left\|(|\tau|+|\xi|)^{\frac{d-1}{4}}\right\| \tau|-| \xi\right|^{-\frac{d-3}{4}} \widetilde{\rho}(\tau, \xi)\left\|_{L^{2}\left(\mathbb{R}^{1+d}\right)} \lesssim\right\| f_{0} \|_{L^{2}\left(\mathbb{R}^{d} \times \mathbb{S}^{d-1}\right)} .
$$

This is slightly better than (1.22) near the cone $\{|\xi|=|\tau|\} \cap\{|\xi| \geq 1\}$ when $d \geq 4$.

Corollary 5.2. Let $d \geq 2$. Under the assumptions and notation of Theorem 4 we have

$$
\left\|(1+|\tau|+|\xi|)^{s}(1+|| \tau|-| \xi||)^{\delta} \widetilde{\rho}(\tau, \xi)\right\|_{L^{2}\left(\mathbb{R}^{1+d}\right)} \lesssim\left\|f_{0}\right\|_{L^{2}\left(\mathbb{R}^{d} \times \mathbb{S}^{d-1}\right)} .
$$

provided that $s+\delta \leq \frac{1}{2}$ and $s \leq \frac{d-1}{4}$.

Proof. Fix $(s, \delta)$ with $s+\delta \leq \frac{1}{2}$ and $s \leq \frac{d-1}{4}$. Define $w_{+}(\tau, \xi)=1+|\xi|+|\tau|$ and $w_{-}(\tau, \xi)=1+|| \xi|-| \tau||$. Then,

$$
w_{+}^{s} w_{-}^{\delta} \leq w_{+}^{s} w_{-}^{\frac{1}{2}-s}=w_{+}^{\frac{d-1}{4}} w_{-}^{-\frac{d-3}{4}}\left(\frac{w_{-}}{w_{+}}\right)^{\frac{d-1}{4}-s} \leq w_{+}^{\frac{d-1}{4}} w_{-}^{-\frac{d-3}{4}}
$$

and the result follows. 


\section{Counterexamples}

In this Section we show that the conditions on $s$ and $\delta$ in Theorem 1 and Corollary 4.2 are necessary. We use the following notation: For $\xi=$ $\left(\xi_{1}, \ldots, \xi_{d}\right) \in \mathbb{R}^{d}$ we set $\xi^{\prime}=\left(\xi_{1}, \ldots, \xi_{d-1}\right)$ and write $\xi=\left(\xi^{\prime}, \xi_{d}\right)$. We denote the Lebesgue measure in $\mathbb{R}^{1+d}$ by $\mu$ and its restriction on $\mathbb{S}^{d-1}$ by $\sigma$.

Proposition 6.1. The conditions (1.11) in Theorem 1 are necessary.

Proof. We prove first that $s+\delta \leq \frac{1}{2}$ is necessary. Fix $N>>1$. Fix $(s, \delta) \in \mathbb{R} \times \mathbb{R}$. Define

$$
\begin{aligned}
& A=\left\{(\tau, \xi) \in \mathbb{R}^{1+d}: 5 \leq \tau \leq 10,\left|\xi^{\prime}\right| \leq 1, N \leq \xi_{d} \leq 2 N\right\} \\
& B=\left\{v \in \mathbb{S}^{d-1}:\left|v_{d}\right| \leq \frac{1}{N}\right\}
\end{aligned}
$$

Define $g \in L^{2}\left(\mathbb{R}^{1+d} \times \mathbb{S}^{d-1}\right)$ by

$$
\tilde{g}(\tau, \xi, v)=\chi_{A}(\tau, \xi) \chi_{B}(v)
$$

Observe that $\sigma(B) \approx \frac{1}{N}\left({ }^{7}\right)$ and

$$
\|g\|_{L^{2}\left(\mathbb{R}^{1+d} \times \mathbb{S}^{d-1}\right)} \approx\|\tilde{g}\|_{L^{2}\left(\mathbb{R}^{1+d} \times \mathbb{S}^{d-1}\right)}=\mu(A)^{\frac{1}{2}} \sigma(B)^{\frac{1}{2}}
$$

For $(\tau, \xi) \in A$ and $v \in B$ we have

$$
|\xi \cdot v| \leq\left|\xi^{\prime}\right|\left|v^{\prime}\right|+\left|\xi_{d}\right|\left|v_{d}\right| \leq 3
$$

and since $5 \leq \tau \leq 10$ we have

$$
\tau+\xi \cdot v \approx 1
$$

Moreover,

$$
1+|\tau|+|\xi| \approx 1+|| \tau|-| \xi|| \approx N
$$

Define $f \in L^{2}\left(\mathbb{R}^{1+d} \times \mathbb{S}^{d-1}\right)$ by

$$
\tilde{f}(\tau, \xi, v)=\frac{\tilde{g}(\tau, \xi, v)}{i(\tau+\xi \cdot v)}
$$

Then $f$ is well defined thanks to (6.4), and satisfies $\partial_{t} f+v \cdot \nabla_{x} f=g$ in the sense of distributions. Also,

$$
|\tilde{f}(\tau, \xi, v)|=\frac{\tilde{g}(\tau, \xi, v)}{\tau+\xi \cdot v} \approx \tilde{g}(\tau, \xi, v)
$$

\footnotetext{
${ }^{7} B$ is a strip of width $\frac{1}{N}$ around the equator.
} 
therefore

$$
\|f\|_{L^{2}\left(\mathbb{R}^{1+d} \times \mathbb{S}^{d-1}\right)}+\|g\|_{L^{2}\left(\mathbb{R}^{1+d} \times \mathbb{S}^{d-1}\right)} \approx\|\tilde{g}\|_{L^{2}\left(\mathbb{R}^{1+d} \times \mathbb{S}^{d-1}\right)}=\mu(A)^{\frac{1}{2}} \sigma(B)^{\frac{1}{2}}
$$

On the other hand

$$
\tilde{\rho}(\tau, \xi)=-i \int_{\mathbb{S}^{d-1}} \frac{\tilde{g}(\tau, \xi, v)}{\tau+\xi \cdot v} d \sigma(v)=-i \chi_{A}(\tau, \xi) \int_{B} \frac{d \sigma(v)}{\tau+\xi \cdot v}
$$

therefore

$$
(1+|\tau|+|\xi|)^{s}(1+|| \tau|-| \xi||)^{\delta}|\tilde{\rho}(\tau, \xi)| \approx N^{s+\delta} \sigma(B) \chi_{A}(\tau, \xi)
$$

which gives

$$
\left\|(1+|\tau|+|\xi|)^{s}(1+|| \tau|-| \xi||)^{\delta}|\tilde{\rho}(\tau, \xi)|\right\|_{L^{2}\left(\mathbb{R}^{1+d} \times \mathbb{S}^{d-1}\right)} \approx N^{s+\delta} \sigma(B) \mu(A)^{\frac{1}{2}}
$$

If estimate (1.12) is true then (6.6) and (6.8) imply that $N^{s+\delta} \sigma(B)^{1 / 2} \lesssim 1$, and since $\sigma(B) \approx \frac{1}{N}$ we have $N^{s+\delta-\frac{1}{2}} \lesssim 1$. Since $N>>1$ was arbitrary we conclude that $s+\delta \leq \frac{1}{2}$.

Next we show that the conditions

$$
\begin{cases}s \leq \min \left\{\frac{d-1}{4}, 1\right\} & \text { if } d \neq 5 \\ s<1 & \text { if } d=5\end{cases}
$$

are necessary. Define

$$
\begin{aligned}
& A=\left\{(\tau, \xi): 10 \leq \tau-|\xi| \leq 20,\left|\xi^{\prime}\right| \leq 1, N \leq \xi_{d} \leq 2 N\right\} \\
& B=\left\{v \in \mathbb{S}^{d-1}:-1+\frac{1}{N} \leq v_{d} \leq 0\right\}
\end{aligned}
$$

For $(\tau, \xi) \in A$ and $v \in B$ we have

$$
\tau+\xi \cdot v=\tau+\xi_{d} v_{d}+\xi^{\prime} \cdot v^{\prime}
$$

and

$$
\tau+\xi_{d} v_{d} \geq \tau+|\xi|\left(-1+\frac{1}{N}\right)=\tau-|\xi|+\frac{|\xi|}{N} \geq \tau-|\xi| \geq 10
$$

while $\left|\xi^{\prime} \cdot v^{\prime}\right| \leq 1$. Therefore

$$
\tau+\xi \cdot v \approx \tau+\xi_{d} v_{d} \gtrsim 1
$$

Moreover

$$
1+|\tau|+|\xi| \approx N, 1+|| \tau|-| \xi|| \approx 1
$$


Define $f, g \in L^{2}\left(\mathbb{R}^{1+d} \times \mathbb{S}^{d-1}\right)$ by

$$
\tilde{g}(\tau, \xi, v)=\frac{\chi_{A}(\tau, \xi) \chi_{B}(v)}{\tau+\xi \cdot v} \quad \tilde{f}(\tau, \xi, v)=\frac{\tilde{g}(\tau, \xi, v)}{i(\tau+\xi \cdot v)}=\frac{\chi_{A}(\tau, \xi) \chi_{B}(v)}{i(\tau+\xi \cdot v)^{2}}
$$

Both $f$ and $g$ are well defined thanks to estimate (6.11) and satisfy $\partial_{t} f+v$. $\nabla_{x} f=g$ in the sense of distibutions. For fixed $(\tau, \xi) \in A$ we have

$$
\int_{\mathbb{S}^{d-1}}|\tilde{g}(\tau, \xi, v)|^{2} d \sigma(v)=\chi_{A}(\tau, \xi) I(\tau, \xi)
$$

where

$$
I(\tau, \xi):=\int_{B} \frac{1}{(\tau+\xi \cdot v)^{2}} d \sigma(v)
$$

Using (6.11) and changing to spherical coordinates ( $\phi$ is the angle between $v$ and $(0, \ldots, 0,1))$ we get

$$
\begin{gathered}
I(\tau, \xi) \approx \int_{B} \frac{1}{\left(\tau+\xi_{d} v_{d}\right)^{2}} d \sigma(v) \approx \int_{\frac{\pi}{2}}^{\arccos \left(-1+\frac{1}{N}\right)} \frac{(\sin \phi)^{d-2}}{\left(\tau+\xi_{d} \cos \phi\right)^{2}} d \phi \\
\quad \stackrel{=-\cos \phi}{=} \int_{0}^{1-\frac{1}{N}} \frac{\left(1-x^{2}\right)^{\frac{d-3}{2}}}{\left(\tau-\xi_{d} x\right)^{2}} d x \approx \int_{0}^{1-\frac{1}{N}} \frac{(1-x)^{\frac{d-3}{2}}}{\left(\tau-\xi_{d} x\right)^{2}} d x
\end{gathered}
$$

Now change variables $x \mapsto t:=\frac{\xi_{d}}{\tau-\xi_{d}}(1-x)$ to get:

$$
I(\tau, \xi) \approx \frac{1}{\left(\tau-\xi_{d}\right)^{2}}\left(\frac{\tau-\xi_{d}}{\xi_{d}}\right)^{\frac{d-1}{2}} \int_{\frac{\xi_{d}}{\tau-\xi_{d}} \frac{1}{N}}^{\frac{\xi_{d}}{\tau-\xi_{d}}} \frac{t^{\frac{d-3}{2}}}{(1+t)^{2}} d t
$$

Recall that $\xi_{d} \approx N,\left|\xi^{\prime}\right| \leq 1$ and $\tau-|\xi| \approx 1$. Therefore $\tau-\xi_{d} \approx 1$, hence

$$
\begin{aligned}
I(\tau, \xi) & \approx \frac{1}{N^{\frac{d-1}{2}}} \int_{1}^{N} \frac{t^{\frac{d-3}{2}}}{(1+t)^{2}} d t \\
& \approx\left\{\begin{array}{ll}
\frac{1}{N^{\min \left\{\frac{d-1}{2}, 2\right\}}} & \text { if } d \neq 5 \\
\frac{\log N}{N^{2}} & \text { if } d=5
\end{array}=: I_{d, N}\right.
\end{aligned}
$$

Therefore

$$
\int_{\mathbb{S}^{d-1}}|\tilde{g}(\tau, \xi, v)|^{2} d \sigma(v) \approx \chi_{A}(\tau, \xi) I_{d, N}
$$

hence

$$
\|g\|_{L^{2}\left(\mathbb{R}^{1+d} \times \mathbb{S}^{d-1}\right)} \approx \mu(A)^{1 / 2} I_{d, N}^{1 / 2}
$$

For $f$ we have, since $\tau+\xi \cdot v \gtrsim 1$ by (6.11), that

$$
|\tilde{f}(\tau, \xi, v)| \lesssim|\tilde{g}(\tau, \xi, v)|
$$


therefore

$$
\|f\|_{L^{2}\left(\mathbb{R}^{1+d} \times \mathbb{S}^{d-1}\right)}+\|g\|_{L^{2}\left(\mathbb{R}^{1+d} \times \mathbb{S}^{d-1}\right)} \approx\|g\|_{L^{2}\left(\mathbb{R}^{1+d} \times \mathbb{S}^{d-1}\right)} \approx \mu(A)^{1 / 2} I_{d, N}^{1 / 2}
$$

For the average $\rho$ we have:

$$
\tilde{\rho}(\tau, \xi)=-i \chi_{A}(\tau, \xi) I(\tau, \xi)
$$

therefore

$$
|\tilde{\rho}(\tau, \xi)| \approx \chi_{A}(\tau, \xi) I_{d, N}
$$

therefore

$$
\left\|(1+|\tau|+|\xi|)^{s}(1+|| \tau|-| \xi||)^{\delta} \tilde{\rho}(\tau, \xi)\right\|_{L^{2}\left(R^{1+d}\right)} \approx N^{s} \mu(A)^{1 / 2} I_{d, N}
$$

If estimate (1.12) is true then

$$
N^{s} \mu(A)^{1 / 2} I_{d, N} \lesssim \mu(A)^{1 / 2} I_{d, N}^{1 / 2}
$$

hence

$$
N^{s} I_{d, N}^{1 / 2} \lesssim 1
$$

If $d \neq 5$ this gives

$$
N^{s-\min }\left\{\frac{d-1}{4}, 1\right\} \lesssim 1
$$

therefore $s \leq \min \left\{\frac{d-1}{4}, 1\right\}$. If $d=5$ then

$$
N^{s-1}(\log N)^{1 / 2} \lesssim 1
$$

therefore $s<1$.

Proposition 6.2. The conditions (4.13) in Corollary 4.2 are necessary.

Proof. We show first that $6 s+2 \delta \leq 1$ is necessary. The construction is similar to those used in Proposition 6.1 but because we need to integrate by parts we replace the characteristic function $\chi_{B}(v)$ by a smooth cut-off function $\phi(v)$. Recall that we are working in $2+1$ dimensions. Given a non-zero vector $\xi \in \mathbb{R}^{2}$ we denote by $\arg \xi$ the angle in $[0,2 \pi)$ formed by $\xi$ and $(1,0)$. For $v \in \mathbb{S}^{1}$ we write $v=(\cos \theta, \sin \theta)$ with $\theta \in[0,2 \pi)$. Then $\Omega_{v}^{1,2}=v_{1} \partial_{v_{2}}-v_{2} \partial_{v_{1}}=\frac{\partial}{\partial \theta}$. For $\xi \in \mathbb{R}^{2} \backslash\{0\}$ and $v \in \mathbb{S}^{d-1}$ we denote by $\measuredangle(\xi, v)$ the angle in $[0, \pi]$ between these two vectors.

Fix $N>>1$. Define $\alpha_{k}=\arcsin \frac{k}{N^{1 / 3}}(k \in \mathbb{N})$. Define

$$
A=\left\{(\tau, \xi): N \leq|\xi| \leq 2 N, 0 \leq \arg \xi \leq \alpha_{5}, N^{1 / 3} \leq \tau-|\xi| \leq 2 N^{1 / 3}\right\}
$$

Choose a smooth cut-off function $\phi: \mathbb{S}^{1} \rightarrow[0,1]$ such that

$$
\begin{aligned}
& \phi(v)=\phi(\cos \theta, \sin \theta)=1 \text { when } \pi-\alpha_{4} \leq \theta \leq \pi-\alpha_{2} \\
& \phi(v)=\phi(\cos \theta, \sin \theta)=0 \text { when } 0 \leq \theta \leq \pi-\alpha_{5} \text { or } 2 \pi \geq \theta \geq \pi-\alpha_{1} \\
& \left|\Omega_{v}^{1,2} \phi(v)\right|=\left|\partial_{\theta}[\phi(\cos \theta, \sin \theta)]\right| \lesssim N^{1 / 3}
\end{aligned}
$$


Observe that

$$
\sigma(\operatorname{supp} \phi) \approx \frac{1}{N^{1 / 3}}
$$

For $(\tau, \xi) \in A$ and $v \in \operatorname{supp} \phi$ we have

$$
\pi-2 \alpha_{5} \leq \measuredangle(\xi, v) \leq \pi-\alpha_{1}
$$

therefore

$$
1-\cos \alpha_{1} \leq 1+\cos \measuredangle(\xi, v) \leq 1-\cos \left(2 \alpha_{5}\right)
$$

Now $1-\cos \alpha_{1} \approx \frac{1}{N^{2 / 3}}$ and $1-\cos \left(2 \alpha_{5}\right) \approx \frac{1}{N^{2 / 3}}$ therefore $1+\cos \measuredangle(\xi, v) \approx$ $\frac{1}{N^{2 / 3}}$. Hence

$$
\tau+\xi \cdot v=\tau-|\xi|+|\xi|(1+\cos \measuredangle(\xi, v)) \approx N^{1 / 3}+N \frac{1}{N^{2 / 3}} \approx N^{1 / 3}
$$

From $(6.22)$ we also get $\sin \measuredangle(\xi, v) \approx \frac{1}{N^{1 / 3}}$, therefore

$$
v_{2} \xi_{1}-v_{1} \xi_{2}=|\xi| \sin \measuredangle(\xi, v) \approx N^{2 / 3}
$$

We define $f, g \in L^{2}\left(\mathbb{R}^{1+2} \times \mathbb{S}^{1}\right)$ by

$$
\tilde{g}(\tau, \xi, v)=\chi_{A}(\tau, \xi) \phi(v) \text { and } \tilde{f}(\tau, \xi, v)=\frac{\Omega_{v}^{1,2} \tilde{g}(\tau, \xi, v)}{i(\tau+\xi \cdot v)}
$$

Thanks to (6.23) they are well defined and satisfy $\partial_{t} f+v \cdot \nabla_{x} f=\Omega_{v}^{1,2} g$ in the sense of distributions. Integrating by parts we find that the corresponding average is given by:

$$
\begin{aligned}
\tilde{\rho}(\tau, \xi) & =\int_{\mathbb{S}^{1}} \tilde{f}(\tau, \xi, v) d \sigma(v)=-i \chi_{A}(\tau, \xi) \int_{\mathbb{S}^{1}} \frac{\Omega_{v}^{1,2} \phi(v)}{\tau+\xi \cdot v} d \sigma(v) \\
& =i \chi_{A}(\tau, \xi) \int_{\mathbb{S}^{1}} \phi(v) \frac{v_{2} \xi_{1}-v_{1} \xi_{2}}{(\tau+\xi \cdot v)^{2}} d \sigma(v)
\end{aligned}
$$

and using $(6.23),(6.24)$ and the fact that $\int_{\mathbb{S}^{1}} \phi(v) d \sigma(v) \approx \frac{1}{N^{1 / 3}}$ we obtain,

$$
|\tilde{\rho}(\tau, \xi)| \approx \frac{\chi_{A}(\tau, \xi)}{N^{1 / 3}}
$$

therefore

$$
\left\|(1+|\tau|+|\xi|)^{s}(1+|| \tau|-| \xi||)^{\delta} \tilde{\rho}(\tau, \xi)\right\|_{L^{2}\left(\mathbb{R}^{1+d}\right)} \approx N^{s+\frac{\delta}{3}-\frac{1}{3}} \mu(A)^{1 / 2}
$$

On the other hand

$$
\tilde{f}(\tau, \xi, v)=\frac{-i \chi_{A}(\tau, \xi) \Omega_{v}^{1,2} \phi(v)}{\tau+\xi \cdot v}
$$

and using (6.21c), (6.23) and (6.21d) we find

$$
\|f\|_{L^{2}\left(\mathbb{R}^{1+2} \times \mathbb{S}^{1}\right)} \lesssim \mu(A)^{1 / 2} \sigma(\operatorname{supp} \phi)^{1 / 2} \approx \mu(A)^{1 / 2} N^{-1 / 6}
$$


Also,

$$
\|g\|_{L^{2}\left(\mathbb{R}^{1+2} \times \mathbb{S}^{1}\right)} \leq \mu(A)^{1 / 2} \sigma(\operatorname{supp} \phi)^{1 / 2} \lesssim \mu(A)^{1 / 2} N^{-1 / 6}
$$

If estimate (4.12) is true then $N^{s+\frac{\delta}{3}-\frac{1}{3}} \lesssim \frac{1}{N^{1 / 6}}$ and since $N$ was an arbitrarily large positive integer this implies $6 s+2 \delta \leq 1$.

To see that $s \leq \frac{1}{6}$ is necessary replace the set $A$ in (6.20) by

$$
A=\left\{(\tau, \xi): N \leq|\xi| \leq 2 N, 0 \leq \arg \xi \leq \alpha_{5}, 1 \leq \tau-|\xi| \leq 2\right\}
$$

Then (6.23) becomes

$$
\tau+\xi \cdot v=\tau-|\xi|+|\xi|(1+\cos \measuredangle(\xi, v)) \approx 1+N \frac{1}{N^{2 / 3}} \approx N^{1 / 3}
$$

and since now $1+|| \tau|-| \xi|| \approx 1,(6.27)$ becomes

$$
\left\|(1+|\tau|+|\xi|)^{s}(1+|| \tau|-| \xi||)^{\delta} \tilde{\rho}(\tau, \xi)\right\|_{L^{2}\left(\mathbb{R}^{1+d} \times \mathbb{S}^{1}\right)} \approx N^{s-\frac{1}{3}} \mu(A)^{1 / 2}
$$

Everything else is exactly the same as before and we get $N^{s-\frac{1}{3}} \lesssim \frac{1}{N^{1 / 6}}$ i.e. $s \leq \frac{1}{6}$.

Finally we show that the condition $s+\delta \leq \frac{1}{4}$ is necessary. Fix $N>>1$. Define new angles $\alpha_{k}$ by $\alpha_{k}=\arccos \left(1-\frac{k}{N}\right)(k \in \mathbb{N})$ and a new set $A$ by

$$
A=\left\{(\tau, \xi): 1 \leq \tau \leq 2, N \leq|\xi| \leq 2 N, 0 \leq \arg \xi \leq \alpha_{5}\right\}
$$

and choose a cut-off function $\phi: \mathbb{S}^{1} \rightarrow[0,1]$ such that

(6.28a) $\phi(v)=\phi(\cos \theta, \sin \theta)=1$ when $\frac{\pi}{2}-\alpha_{4} \leq \theta \leq \frac{\pi}{2}-\alpha_{2}$

(6.28b) $\phi(v)=\phi(\cos \theta, \sin \theta)=0$ when $0 \leq \theta \leq \frac{\pi}{2}-\alpha_{5}$ or $2 \pi \geq \theta \geq \frac{\pi}{2}-\alpha_{1}$

(6.28c) $\quad\left|\Omega_{v}^{1,2} \phi(v)\right|=\left|\partial_{\theta} \phi(\cos \theta, \sin \theta)\right| \lesssim N^{1 / 2}$

Observe that

$$
\sigma(\operatorname{supp} \phi) \approx \frac{1}{N^{1 / 2}}
$$

For $(\tau, \xi) \in A$ and $v \in \operatorname{supp} \phi$ we have

$$
\frac{\pi}{2}-2 \alpha_{5} \leq \measuredangle(\xi, v) \leq \frac{\pi}{2}-\alpha_{1}
$$

therefore

Thus

$$
\cos \measuredangle(\xi, v) \approx \frac{1}{N^{1 / 2}} \quad, \quad \sin \measuredangle(\xi, v) \approx 1
$$

and

$$
\tau+\xi \cdot v=\tau+|\xi| \cos \measuredangle(\xi, v) \approx 1+N \frac{1}{N^{1 / 2}} \approx N^{1 / 2}
$$

$$
v_{2} \xi_{1}-v_{1} \xi_{2}=|\xi| \sin \measuredangle(\xi, v) \approx N
$$


Define $f$ and $g$ as in (6.25). Using (6.26) and $\int_{\mathbb{S}^{1}} \phi(v) d \sigma(v) \approx \frac{1}{N^{1 / 2}}$ we find

therefore

$$
|\tilde{\rho}(\tau, \xi)| \approx \frac{\chi_{A}(\tau, \xi)}{N^{1 / 2}}
$$

$$
\left\|(1+|\tau|+|\xi|)^{s}(1+|| \tau|-| \xi||)^{\delta} \tilde{\rho}(\tau, \xi)\right\|_{L^{2}\left(\mathbb{R}^{1+d}\right)} \approx \mu(A)^{1 / 2} N^{s+\delta-\frac{1}{2}}
$$

On the other hand it is easy to see that

$$
\|f\|_{L^{2}\left(\mathbb{R}^{1+2} \times \mathbb{S}^{1}\right)}+\|g\|_{L^{2}\left(\mathbb{R}^{1+2} \times \mathbb{S}^{1}\right)} \lesssim \mu(A)^{1 / 2} \sigma(\operatorname{supp} \phi)^{1 / 2} \approx \mu(A)^{1 / 2} N^{-1 / 4}
$$

If estimate $(4.12)$ is true then $N^{s+\delta-\frac{1}{2}} \lesssim N^{-\frac{1}{4}}$ and since $N$ was arbitrary, $s+\delta \leq \frac{1}{4}$

Proposition 6.3. Let $d \geq 2$. The conditions $s+\delta \leq \frac{1}{2}$ and $s \leq \frac{d-1}{4}$ in Corollary 5.2 are necessary.

Proof. First we show the necessity of the condition $s \leq(d-1) / 4$. Fix $N>>1$ and consider the following sets:

$$
A=\left\{\xi \in \mathbb{R}^{d}: N \leq|\xi| \leq 2 N\right\}
$$

and

$$
B=\left\{v \in \mathbb{S}^{d-1}: v=(\omega \sin \theta, \cos \theta), \frac{1}{4 N^{1 / 2}} \leq \theta \leq \frac{1}{2 N^{1 / 2}}, \omega \in \mathbb{S}^{d-2}\right\} .
$$

Define $f_{0}$ by

$$
\widehat{f_{0}}(\xi, v)=\chi_{A}(\xi) \chi_{B}(v)
$$

Define

$$
D_{\xi, N}=\left[-|\xi| \cos \frac{1}{4 N^{1 / 2}},-|\xi| \cos \frac{1}{2 N^{1 / 2}}\right]
$$

We show that

$$
\tilde{\rho}(\tau, \xi)=c(d) \frac{1}{|\xi|}\left(1-\frac{\tau^{2}}{|\xi|^{2}}\right)^{\frac{d-3}{2}} \chi_{A}(\xi) \chi_{D_{\xi, N}}(\tau)
$$

Suppose $d \geq 3$. Using (5.6) we have

$$
\tilde{\rho}(\tau, \xi)=\frac{1}{|\xi|}\left(1-\frac{\tau^{2}}{|\xi|^{2}}\right)^{\frac{d-3}{2}} \int_{\mathbb{S}^{d-2}} \widehat{f}_{0}\left(\xi,\left(1-\frac{\tau^{2}}{|\xi|^{2}}\right)^{1 / 2} \omega,-\frac{\tau}{|\xi|}\right) d \sigma(\omega)
$$

$$
=\frac{1}{|\xi|}\left(1-\frac{\tau^{2}}{|\xi|^{2}}\right)^{\frac{d-3}{2}} \chi_{A}(\xi) \int_{\mathbb{S}^{d-2}} \chi_{B}\left(\left(1-\frac{\tau^{2}}{|\xi|^{2}}\right)^{1 / 2} \omega,-\frac{\tau}{|\xi|}\right) d \sigma(\omega)
$$


Fix $\xi \in A$. It is easy to check that if $\tau \in D_{\xi, N}$ then for all $\omega \in \mathbb{S}^{d-2}$ we have $\left(\left(1-\frac{\tau^{2}}{|\xi|^{2}}\right)^{1 / 2} \omega,-\frac{\tau}{|\xi|}\right) \in B$, and therefore the integrand in (6.34) is identically equal to one. On the other hand, if $\tau \notin D_{\xi, N}$ then for all $\omega \in \mathbb{S}^{d-2}$ we have $\left(\left(1-\frac{\tau^{2}}{|\xi|^{2}}\right)^{1 / 2} \omega,-\frac{\tau}{|\xi|}\right) \notin B$, and therefore, the integrand in (6.34) is identically equal to zero. This proves (6.32) when $d \geq 3$. If $d=2$ we replace (6.33) by (see Section 5 of [6])

$$
\tilde{\rho}(\tau, \xi)=\frac{1}{|\xi|}\left(1-\frac{\tau^{2}}{|\xi|^{2}}\right)^{-\frac{1}{2}} \widehat{f_{0}}\left(\xi,\left(1-\frac{\tau^{2}}{|\xi|^{2}}\right)^{1 / 2},-\frac{\tau}{|\xi|}\right)
$$

and work similarly. It follows from (6.32) that

$$
\operatorname{supp} \tilde{\rho}=\left\{(\tau, \xi): \xi \in A,-|\xi| \cos \frac{1}{4 N^{1 / 2}} \leq \tau \leq-|\xi| \cos \frac{1}{2 N^{1 / 2}}\right\}
$$

therefore, for $(\tau, \xi) \in \operatorname{supp} \tilde{\rho}$, we have $|\xi| \approx N$ and $\tau \approx-N$ hence $|\tau|+|\xi| \approx N$. Also,

$$
-|\xi|\left(1-\frac{c}{N}\right) \leq-|\xi| \cos \frac{1}{4 N^{1 / 2}} \leq \tau \leq-|\xi| \cos \frac{1}{2 N^{1 / 2}} \leq-|\xi|\left(1-\frac{c^{\prime}}{N}\right)
$$

therefore

$$
|| \xi|-| \tau||=|\xi|+\tau \approx|\xi| \frac{1}{N} \approx 1
$$

It follows that

$$
\tilde{\rho}(\tau, \xi)=\frac{(|\xi|-|\tau|)^{\frac{d-3}{2}}(|\xi|+|\tau|)^{\frac{d-3}{2}}}{|\xi|^{d-2}} \chi_{A}(\xi) \chi_{D_{\xi, N}}(\tau) \approx N^{-\frac{d-1}{2}} \chi_{A}(\xi) \chi_{D_{\xi, N}}(\tau)
$$

therefore

$$
\begin{aligned}
\left\|(1+|\xi|+|\tau|)^{s}(1+|| \xi|-| \tau||)^{\delta} \tilde{\rho}(\tau, \xi)\right\|_{L^{2}\left(\mathbb{R}^{1+d}\right)}^{2} & \int_{\mathbb{R}^{d}} \int_{\mathbb{R}} \chi_{A}(\xi) \chi_{D_{\xi, N}}(\tau) d \tau d \xi \\
& \approx N^{2 s-(d-1)} \int_{A}|\xi|\left(\cos \frac{1}{4 N^{1 / 2}}-\cos \frac{1}{2 N^{1 / 2}}\right) d \xi \\
& =N^{2 s-(d-1)} \int^{2 s-(d-1)} \mu(A)
\end{aligned}
$$

On the other hand,

$$
\left\|f_{0}\right\|_{L^{2}\left(\mathbb{R}^{d} \times \mathbb{S}^{d-1}\right)}^{2}=\mu(A) \sigma(B) \approx \frac{\mu(A)}{N^{\frac{d-1}{2}}} .
$$

We conclude that in order for the estimate (5.13) to be true it is necessary that $N^{2 s-(d-1)} \lesssim N^{-\frac{d-1}{2}}$ for arbitrarily large $N$. Thus, $s \leq(d-1) / 4$. 
Next we show that $s+\delta \leq \frac{1}{2}$ is necessary. We define $A$ as in (6.29), define a new set $B$ by

$$
B=\left\{v \in \mathbb{S}^{d-2}: v=(\omega \sin \theta, \cos \theta), \frac{\pi}{2}+\frac{1}{N} \leq \theta \leq \frac{\pi}{2}+\frac{2}{N}, \omega \in \mathbb{S}^{d-2}\right\}
$$

and a new set $D_{\xi, N}$ by

$$
D_{\xi, N}=\left[|\xi| \sin \frac{1}{N},|\xi| \sin \frac{2}{N}\right]
$$

We define $f_{0}$ by (6.31). Working as above we can show that

$$
\tilde{\rho}(\tau, \xi)=c(d) \frac{1}{|\xi|}\left(1-\frac{\tau^{2}}{|\xi|^{2}}\right)^{\frac{d-3}{2}} \chi_{A}(\xi) \chi_{D_{\xi, N}}(\tau)
$$

Therefore, on the support of $\tilde{\rho}$ we have $|\xi| \approx N$ and $\tau \approx 1$, hence $|\tau|+|\xi| \approx N$ and ||$\tau|-| \xi|| \approx N$. This gives $\tilde{\rho}(\tau, \xi) \approx \frac{1}{N} \chi_{A}(\xi) \chi_{D_{\xi, N}}(\tau)$, and working as in the proof of $(6.37)$ we get

$$
\left\|(1+|\xi|+|\tau|)^{s}(1+|| \xi|-| \tau||)^{\delta} \tilde{\rho}(\tau, \xi)\right\|_{L^{2}\left(\mathbb{R}^{1+d}\right)}^{2} \approx N^{2 s+2 \delta-2} \mu(A)
$$

On the other hand

$$
\left\|f_{0}\right\|_{L^{2}\left(\mathbb{R}^{d} \times \mathbb{S}^{d-1}\right)}^{2}=\mu(A) \sigma(B) \approx \frac{\mu(A)}{N}
$$

These two estimates give $N^{2 s+2 \delta-2} \lesssim N^{-1}$, hence $s+\delta \leq \frac{1}{2}$.

\section{References}

[1] Bardos, C., Golse, F., Perthame, B.: The Rosseland approximation for the radiative transfer equations. Comm. Pure Appl. Math. 40 (1987), no. $6,691-721$.

[2] Bardos, C., Golse, F., Perthame, B., Sentis, R.: The nonaccretive radiative transfer equations: existence of solutions and Rosseland approximation. J. Funct. Anal. 77 (1988), no. 2, 434-460.

[3] Bouchut, F.: Introduction to the mathematical theory of kinetic equations. In Kinetic Equations and Asymptotic Theories. Series in Appl. Math. 4. Gauthier-Villars, Éditions Scientifiques et Médicales Elsevier, Paris, 2000.

[4] Bouchut, F., Desvillettes, L.: Averaging Lemmas without time Fourier Transform and applications to discretized kinetic equations. Proc. Roy. Soc. Edinburgh, Sect. A 129 (1999), no. 1, 19-36. 
[5] Bourgain, J.: Fourier Transform Restriction phenomena for certain lattice subsets and applications to nonlinear evolution equations. Part I: Geom. Funct. Anal. 3 (1993), no. 2, 107-156. Part II: Geom. Funct. Anal. 3 (1993), no. 3, 209-262.

[6] Bournaveas, N., Perthame, B.: Averages over spheres for kinetic transport equations: hyperbolic Sobolev spaces and Strichartz inequalities. J. Math. Pures Appl. 80 (2001), no. 5, 517-534.

[7] Castella, F., Perthame, B.: Estimations de Strichartz pour les équations de transport cinétique. C.R. Acad. Sci. Paris Sér. I Math. 322 (1996), no. 6, 535-540.

[8] Chandrasekhar, S.: Radiative transfer. Oxford University Press, 1950.

[9] Desvillettes, L., Mischler, S.: About the splitting algorithm for Boltzmann and B.G.K. equations. Math. Models Methods Appl. Sci. 6 (1996), no. 8, 1079-1101.

[10] DeVore, R., Petrova, G.: The averaging lemma. J. Amer. Math. Soc. 14 (2001), no. 2, 279-296.

[11] DiPerna, R. J., Lions, P. L.: On the Cauchy problem for Boltzmann equations: global existence and weak stability results. Ann. of Math. (2) 130 (1989), no. 2, 321-366.

[12] DiPerna, R. J., Lions, P. L.: Global weak solutions of Vlasov-Maxwell systems. Comm. Pure Appl. Math. 42 (1989), no. 6, 729-757.

[13] DiPerna, R. J., Lions, P. L., Meyer, Y.: $L^{p}$ regularity of velocity averages. Ann. Inst. H. Poincaré, Anal. Non Linéaire 8 (1991), no. 3-4, 271-287.

[14] Foschi, D., Klainerman, S.: Bilinear space-time estimates for homogeneous wave equations. Ann. Sci. École Norm. Sup. (4) 33, (2000), no. 2, 211-274.

[15] GÉrard, P.: Microlocal defect measures. Comm. Partial Differential Equations 16 (1991), no. 11, 1761-1794.

[16] Golse, F., Perthame, B.: Generalized solutions of the radiative transfer equations in a singular case. Comm. Math. Phys. 106 (1986), no. 2, 211-239.

[17] Golse, F., Lions, P. L., Perthame, B., Sentis, R.: Regularity of the moments of the solution of a transport equation. J. Funct. Anal. 76 (1988), no. $1,110-125$.

[18] Golse, F., Perthame, B., Sentis, R.: Un résultat de compacité pour les équations du transport et application au calcul de la limite de la valeur propre principale d'un opérateur de transport. C. R. Acad. Sci. Série I Math. 301 (1985), no. 7, 341-344.

[19] Jabin, P.-E., VEGA, L.: A real space method for averaging lemmas. J. Math. Pures Appl. (9) 83 (2004), no. 11, 1309-1351.

[20] Jabin, P.-E., Vega, L.: Averaging lemmas and the X-ray transform. C. R. Math. Acad. Sci. Paris 337 (2003), no. 8, 505-510. 
[21] Jabin, P.-E., Perthame, B.: Regularity in kinetic formulations via averaging lemmas. ESAIM Control Optim. Calc. Var. 8 (2002), 761-774.

[22] Lions, P. L., Perthame, B.: Lemmes de moments, de moyenne et de dispersion. C. R. Acad. Sci. Paris Sér. I Math. 314 (1992), no. 11, 801-806.

[23] Lions, P. L.: Régularité optimale des moyennes en vitesses. C. R. Acad. Sci. Sér. I Math. 320 (1995), no. 8, 911-915.

[24] Perthame, B.: Mathematical tools for kinetic equations. Bull. Amer. Math. Soc. (N.S.) 41 (2004), no. 2, 205-244.

[25] Perthame, B., VÁzquez, J. L.: Bounded speed of propagation for solutions to radiative transfer equations. Comm. Math. Phys. 130 (1990), no. 3, 457-469.

[26] Perthame, B., Souganidis, P. E.: A limiting case for velocity averaging. Ann. Sci. École Norm. Sup. (4) 31 (1998), no. 4, 591-598.

[27] Tataru, D.: The $X_{\theta}^{s}$ spaces and unique continuation for solutions to the semilinear wave equation. Comm. Partial Differential Equations 21 (1996), no. 5-6, 841-887.

Recibido: 28 de abril de 2005

Nikolaos Bournaveas University of Edinburgh School of Mathematics JCMB, King's Buildings Edinburgh EH9 3JZ, UK N.Bournaveas@ed.ac.uk

Susana Gutiérrez École Normale Supérieure Département de mathématiques et applications 45 rue d'Ulm, F 75230

Paris, cedex 05, France Susana.Gutierrez@ens.fr

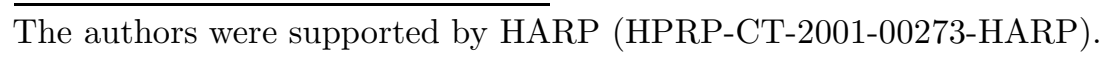

